\title{
CONNECTIONS WITH EXOTIC HOLONOMY
}

\author{
LORENZ J. SCHWACHHÖFER
}

\begin{abstract}
Berger [Ber] partially classified the possible irreducible holonomy representations of torsion free connections on the tangent bundle of a manifold. However, it was shown by Bryant [Bry] that Berger's list is incomplete. Connections whose holonomy is not contained on Berger's list are called exotic.

We investigate a certain 4-dimensional exotic holonomy representation of $S l(2, \mathbb{R})$. We show that connections with this holonomy are never complete and do not exist on compact manifolds. We give explicit descriptions of these connections on an open dense set and compute their groups of symmetry.
\end{abstract}

\section{INTRODUCTION}

Let $M^{n}$ be a smooth connected $n$-dimensional manifold. Let $\mathscr{P}(M)$ denote the set of piecewise smooth paths $\gamma:[0,1] \rightarrow M$, and for $x \in M$, let $\mathscr{L}_{x}(M) \subseteq$ $\mathscr{P}(M)$ denote the set of $x$-based loops, i.e. paths for which $\gamma(0)=\gamma(1)=x$.

Let $\nabla$ be a torsion free affine connection on the tangent bundle of $M$. For each $\gamma \in \mathscr{P}(M)$, the connection $\nabla$ defines a linear isomorphism $P_{\gamma}: T_{\gamma(0)} M \rightarrow$ $T_{\gamma(1)} M$, called parallel translation along $\gamma$. For each $x \in M$, we define the holonomy group of $\nabla$ at $x$ to be $H_{x}:=\left\{P_{\gamma} \mid \gamma \in \mathscr{L}_{x}\right\} \subseteq G l\left(T_{x} M\right)$.

It is well known that $H_{x}$ is a Lie subgroup of $G l\left(T_{x} M\right)$, and that for any $\gamma \in$ $\mathscr{P}(M), P_{\gamma}$ induces an isomorphism of $T_{\gamma(0)} M$ with $T_{\gamma(1)} M$ which identifies $H_{\gamma(0)}$ with $H_{\gamma(1)}[\mathrm{KN}]$.

Choose an $x_{0} \in M$ and an isomorphism $i: T_{x_{0}} M \rightarrow \mathbb{R}^{n}$. Then, because $M$ is connected, the conjugacy class of the subgroup $H \subseteq G l(n, \mathbb{R})$ which corresponds under $i$ to $H_{x_{0}} \subseteq G l\left(T_{x_{0}} M\right)$ is independent of the choice of $x_{0}$ or $i$. By abuse of language, we speak of $H$ as the holonomy group and of the Lie algebra $\mathfrak{h}$ of $H$ as the holonomy algebra of $(M, \nabla)$.

The following is a basic question in the theory:

Which (conjugacy classes of) subgroups $H \subseteq G l(n, \mathbb{R})$ can occur as the holonomy of some torsion free connection $\nabla$ on some n-manifold $M$ ?

The condition of torsion freeness makes this problem nontrivial. In fact, it is not hard to see that any representation of a connected Lie group can be realized as the holonomy of some connection (with torsion) on some manifold.

A necessary condition on the holonomy algebra of a torsion free connection was derived by M. Berger [Ber] in his thesis as follows.

Received by the editors April 1, 1993 and, in revised form, September 22, 1993.

1991 Mathematics Subject Classification. Primary 53A15; Secondary 53B05. 
Let $V$ be a vector space and define for a given Lie algebra $\mathfrak{g} \subseteq \mathfrak{g} l(V)$ :

$$
\mathbf{K}(\mathfrak{g}):=\left\{\varphi \in \Lambda^{2}\left(V^{*}\right) \otimes \mathfrak{g} \mid \sum_{\sigma \in A_{3}} \varphi\left(u_{\sigma(1)}, u_{\sigma(2)}\right) u_{\sigma(3)}=0 \text { for } u_{1}, u_{2}, u_{3} \in V\right\},
$$

and

$$
\mathbf{K}^{1}(\mathfrak{g}):=\left\{\psi \in V^{*} \otimes \mathbf{K}(\mathfrak{g}) \mid \sum_{\sigma \in A_{3}} \psi\left(u_{\sigma(1)}\right)\left(u_{\sigma(2)}, u_{\sigma(3)}\right)=0 \text { for } u_{1}, u_{2}, u_{3} \in V\right\} .
$$

Given $(M, \nabla)$ as above and $x_{0} \in M$, the curvature tensor of $\nabla$ at $x_{0}$, i.e. the map $R_{x_{0}}: \Lambda^{2}\left(T_{x_{0}} M\right) \rightarrow \mathfrak{g l}\left(T_{x_{0}} M\right)$ defined by $R_{x_{0}}(u, v) w=\nabla_{u} \nabla_{v} w-$ $\nabla_{v} \nabla_{u} w-\nabla_{[u, v]} w$, is known to have its values in the holonomy algebra $\mathfrak{h}_{x_{0}} \subseteq$ $\mathfrak{g l}\left(T_{x_{0}} M\right)$, and to satisfy the first and second Bianchi identities. This is equivalent to saying

$$
R_{x_{0}} \in \mathbf{K}\left(\mathfrak{h}_{x_{0}}\right), \quad \text { and } \nabla R_{x_{0}} \in \mathbf{K}^{1}\left(\mathfrak{h}_{x_{0}}\right) .
$$

In this notation, Berger's criterion is:

$$
\begin{aligned}
& \text { If } \mathfrak{g}^{\prime} \subsetneq \mathfrak{g} \text { is a proper subalgebra, and } \mathbf{K}\left(\mathfrak{g}^{\prime}\right)=\mathbf{K}(\mathfrak{g}) \text {, then } \mathfrak{g} \\
& \text { cannot be the holonomy algebra of any torsion free connection } \\
& \text { on any } n \text {-manifold } M \text {. }
\end{aligned}
$$

This criterion is a consequence of the Ambrose-Singer Holonomy Theorem, which states that the holonomy algebra $\mathfrak{h}_{x_{0}}$ is generated by the image of the curvature map $R_{x_{0}}$ and its parallel translations [KN, II.8.1].

The study of locally symmetric connections, i.e. connections with $\nabla R=0$, can be reduced to certain problems in the theory of Lie algebras. We therefore wish to exclude this case from our discussion. A second necessary condition for $\mathfrak{g}$ to be the holonomy of a torsion free connection which is not locally symmetric is therefore $\mathbf{K}^{\mathbf{1}}(\mathfrak{g}) \neq 0$.

These two criteria are also referred to as Berger's first and second criterion. Using these, Berger [Ber] was able to partially classify the possible Lie algebras of holonomy groups of torsion free connections which are not locally symmetric. His classification falls into three parts:

(1) The first part classifies all possible Riemannian holonomies, i.e. connections with holonomy group $H \subseteq O(n, \mathbb{R})$. It turns out that the possible holonomy groups of nonsymmetric connections are those subgroups which act transitively on the unit sphere in $\mathbb{R}^{n}$. In fact, it is by now well known which elements in Berger's list actually do occur as holonomies of Riemannian metrics. We mention in this context the work of Simons [S], Calabi [C], Alekseevskii [A] and Bryant [Br1].

(2) The second part classifies all possible irreducible pseudo-Riemannian holonomies, i.e. connections with holonomy group $H \subseteq O(p, q)$. In this case, the question whether or not these candidates actually do occur as holonomies has been resolved except for the group $S O^{*}(2 n) \subseteq$ $G l(4 n, \mathbb{R})$ for $n \geq 3$.

(3) The third part classifies the possible irreducibly acting holonomy groups of affine torsion free connections, i.e. holonomy groups which do not leave invariant any nondegenerate symmetric bilinear form. These connections are the least understood. In fact, Berger's list in this case is 
incomplete, conceivably omitting a finite number of possibilities. The holonomies which are not contained in Berger's list are referred to as exotic holonomies.

R. Bryant [Br2] showed that exotic holonomies do, in fact, exist. He investigated the irreducible representations of $S l(2, \mathbb{R})$ which can be described as follows:

For $n \in \mathbb{N}$, let $V_{n}:=\{$ homogeneous polynomials in $x$ and $y$ of degree $n$ \} which is an $n+1$-dimensional vector space. There is an $S l(2, \mathbb{R})$-action on $V_{n}$ induced by the transposed action of $\operatorname{Sl}(2, \mathbb{R})$ on $\mathbb{R}^{2}$, i.e. if $p \in V_{n}$ and $A \in \operatorname{Sl}(2, \mathbb{R})$ then

$$
(A \cdot p)(x, y):=p(u, v) \quad \text { with }(u, v)(x, y) A .
$$

It is well known that this action is irreducible for every $n$ and moreover that - up to equivalence-this is the only irreducible $n+1$-dimensional representation of $S l(2, \mathbb{R})[\mathrm{BD}]$.

Let $H_{n} \subseteq G l\left(V_{n}\right)$ be the image of this representation and let $\mathfrak{h}_{n} \subseteq \mathfrak{g} l\left(V_{n}\right)$ be the Lie algebra of $H_{n}$. Bryant showed that $\mathfrak{h}_{n}$ does not satisfy Berger's first and second criterion if $n \geq 4$.

For $n=3$, however, he proved the existence of torsion free connections on 4-manifolds whose holonomy group is $H_{3}$, even though this group does not appear on Berger's list. We shall refer to these connections as $\mathrm{H}_{3}$-connections.

Note that for $n$ odd, there is no nondegenerate symmetric bilinear form which is invariant under the $S l(2, \mathbb{R})$-action on $V_{n}$. There is, however, an invariant 2-form and hence we conclude that any $\mathrm{H}_{3}$-connection must admit a parallel symplectic form.

A diffeomorphism $\varphi: M \rightarrow M$ preserving the connection will be called a symmetry of $\nabla$.

It turns out that locally there are very few examples of $\mathrm{H}_{3}$-connections. In fact, the local classification given by Bryant can be summarized as follows:

(1) There is one example of a homogeneous $\mathrm{H}_{3}$-connection whose symmetry group is 5-dimensional.

(2) There is a finite set of $H_{3}$-connections with a 3-dimensional symmetry group.

(3) There is a 1-parameter family of $\mathrm{H}_{3}$-connections with a 1-dimensional symmetry group.

$H_{3}$-connections with a 1-dimensional symmetry group will be called regular, all others will be called singular $\mathrm{H}_{3}$-connections.

Bryant's classification is obtained by the methods of Exterior Differential Systems. This approach, however, makes a concrete description of these connections very difficult. In this article, we will describe the $H_{3}$-connections more explicitly and will also investigate their global behavior.

(1) The homogeneous $H_{3}$-connection can be described globally.

(2) The singular $\mathrm{H}_{3}$-connections can be described on the dense open subsets on which the action of their symmetry groups is locally free.

(3) For the regular $\mathrm{H}_{3}$-connections we can only give a description on some open subset which will not be dense in general.

A global description of the homogeneous $H_{3}$-connection was given in [Br2]. However, our description includes the symmetry group and presents the 
connection in a somewhat more explicit way. In fact, we will compute the Lie algebras of the symmetry groups of all $\mathrm{H}_{3}$-connections.

Furthermore, in the case of some singular $\mathrm{H}_{3}$-connections we can conclude that the parallel symplectic form must be exact.

Following this introduction, we will first give the descriptions of the singular $H_{3}$-connections $(\S 2)$.

In $\S 3$, we derive the structure equations for $\mathrm{H}_{3}$-connections, following closely [Br2].

As a first global result we will show in $\S 4$ the

Theorem 4.1. $H_{3}$-connections are never complete.

In $\S 5$, we then solve the structure equations for the singular $\mathrm{H}_{3}$-connections under the generic assumption that the group action of the symmetry group is locally free, as well as the structure equations for the regular $\mathrm{H}_{3}$-connections on some open subset. This will show that the examples given in $\S 2$ form in fact a complete list of singular $\mathrm{H}_{3}$-connections under these restrictions.

Finally, in $\S 6$ we shall be concerned with $\mathrm{H}_{3}$-connections on compact manifolds, and we will prove

Theorem 6.1. There are no $\mathrm{H}_{3}$-connections on compact manifolds.

Note that Theorem 6.1 is not a consequence of Theorem 4.1 since affine connections on compact manifolds are not necessarily complete.

The main part of this article represents the author's thesis and he wishes to thank his advisor Wolfgang Ziller for his encouragement and support.

\section{EXAMPLES OF SINGULAR $H_{3}$-CONNECTIONS}

We first introduce some notational conventions. Recall that $V_{3}$ is the vector space of homogeneous polynomials of degree 3 in the variables $x$ and $y$, and the action of $S l(2, \mathbb{R})$ on $V_{3}$ is induced by the transposed action of $S l(2, \mathbb{R})$ on $\mathbb{R}^{2}=\operatorname{span}\{x, y\}$.

We let $\rho_{3}: S l(2, \mathbb{R}) \rightarrow H_{3} \subseteq G l\left(V_{3}\right)$ and $\left(\rho_{3}\right)_{*}: \mathfrak{s l}(2, \mathbb{R}) \rightarrow \mathfrak{h}_{3} \subseteq \mathfrak{g l}\left(V_{3}\right)$ denote the representation homomorphisms, and define a basis $\left\{E_{1}, E_{2}, E_{3}\right\}$ of $\mathfrak{h}_{3}$ by $E_{i}:=\left(\rho_{3}\right)_{*}\left(\tilde{E}_{i}\right)$ where the basis $\left\{\tilde{E}_{1}, \tilde{E}_{2}, \tilde{E}_{3}\right\}$ of $\mathfrak{s l}(2, \mathbb{R})$ is given by

$$
\left(\begin{array}{cc}
a & c \\
b & -a
\end{array}\right)=a \tilde{E}_{1}+b \tilde{E}_{2}+c \tilde{E}_{3} .
$$

Furthermore, we let $\left\{e_{0}, \ldots, e_{3}\right\}$ with $e_{i}=x^{3-i} y^{i}$ be a basis of $V_{3}$, and $\left\{\underline{e}_{i} \mid 0 \leq i \leq 3\right\}$ be the standard basis of $\mathbb{R}^{4}$.

We fix once and for all a linear isomorphism

$$
\begin{aligned}
\lambda: V_{3} & \longrightarrow \mathbb{R}^{4} \\
e_{i} & \longmapsto \underline{e}_{i} .
\end{aligned}
$$

Then $\underline{\mathfrak{h}}_{3}:=\lambda \mathfrak{h}_{3} \lambda^{-1} \subseteq \mathfrak{g l}(4, \mathbb{R})$ has $\left\{\underline{E}_{1}, \underline{E}_{2}, \underline{E}_{3}\right\}$ with $\underline{E}_{i}=\lambda E_{i} \lambda^{-1}$ as a basis, and one sees that

$$
a \underline{E}_{1}+b \underline{E}_{2}+c \underline{E}_{3}\left(\begin{array}{cccc}
3 a & c & & \\
3 b & a & 2 c & \\
& 2 b & -a & 3 c \\
& & b & -3 a
\end{array}\right)
$$


We shall from now on use $\lambda$ to identify $\mathfrak{h}_{3}$ with $\underline{\mathfrak{h}}_{3}$.

Recall that if $M$ is a manifold with an $H_{3}$-connection $\nabla$ then a diffeomorphism $\varphi: M \rightarrow M$ preserving the connection $\nabla$, i.e. satisfying

$$
\varphi_{*}\left(\nabla_{X} Y\right)=\nabla_{\varphi_{*}(X)} \varphi_{*}(Y)
$$

for all vector fields $X$ and $Y$ on $M$, will be called a symmetry of $\nabla$. We shall call a vector field $S$ on $M$ an infinitesimal symmetry iff $\mathfrak{L}_{S} \nabla=0$, i.e.

$$
\left[S, \nabla_{X} Y\right]=\nabla_{[S, X]} Y+\nabla_{X}[S, Y]
$$

for all vector fields $X$ and $Y$ on $M$. Note that the flow along an infinitesimal symmetry is a 1-parameter family of (local) symmetries.

In order to describe a connection on a manifold $M$, we shall in each case give a frame on $M$ and the connection form $\phi$ w.r.t. this frame which takes values in $\mathfrak{h}_{3}$. This form is the pullback of the connection form on the coframe bundle of $M$ under the section given by the frame.

Let $X_{0}, \ldots, X_{3}$ be the given frame and let $\omega_{0}, \ldots, \omega_{3}$ denote the dual coframe. We define the $V_{3}$-valued 1-form $\omega$ by

$$
\omega=\sum_{i} \omega_{i} e_{i}
$$

which establishes an isomorphism between $T_{p} M$ and $V_{3}$ for all $p \in M$.

Then we can describe the covariant derivative associated to the connection by

$$
\omega\left(\nabla_{X_{i}} X_{j}\right):=-\phi\left(X_{i}\right) \cdot \omega\left(X_{j}\right)=-\phi\left(X_{i}\right) \cdot e_{j} .
$$

The connection being torsion free is equivalent to the condition that

$$
\phi\left(X_{i}\right) \cdot e_{j}-\phi\left(X_{j}\right) \cdot e_{i}=d \omega\left(X_{i}, X_{j}\right) \quad \text { for all } i, j .
$$

The holonomy algebra of these connections is contained in $\mathfrak{h}_{3}$ by the AmbroseSinger-Holonomy Theorem mentioned earlier. In fact, we will show (Corollary 3.2) that the holonomy algebra of any such connection is actually equal to $\mathfrak{h}_{3}$, provided the connection is not flat.

As we shall see in $\S 3$, to every $\mathrm{H}_{3}$-connection we can associate a pair of homogeneous polynomials $a \in V_{2}$ and $b \in V_{3}$ as well as a constant $c \in \mathbb{R}$. For future reference we shall give these polynomials, called the structure polynomials, and this constant in each particular case.

The description of these examples will be motivated in $\S 5$.

2.1 Example I : The homogeneous case (type $\left.\Sigma_{0}^{0}\right)$. Let

$$
G=\operatorname{ASl}(2, \mathbb{R})=\left\{\left(\begin{array}{ccc}
A & x \\
0 & 0 & 1
\end{array}\right) \mid A \in \operatorname{Sl}(2, \mathbb{R}), x, y \in \mathbb{R}\right\}
$$

be the group of unimodular affine motions of $\mathbb{R}^{2}$ and let $\mathfrak{g}$ be the Lie algebra of $G$. Let $A_{i j}$ stand for the $3 \times 3$-matrix with $(i, j)$ th entry 1 and all other entries 0 . Then we define a basis of the Lie algebra $\mathfrak{g}$ of $G$ by

$$
\begin{aligned}
& Z_{0}=\frac{9}{4} A_{23}, \\
& Z_{1}=-\frac{3}{2}\left(A_{13}-A_{21}\right), \\
& Z_{2}=-3\left(A_{11}-A_{22}\right), \\
& Z_{3}=-18 A_{12}, \\
& Y=A_{13}+2 A_{21}
\end{aligned}
$$


and the subgroup $H \leq G$ as $H=\{\exp (t Y) \mid t \in \mathbb{R}\}$. Furthermore, we decompose $\mathfrak{g}$ as $\mathfrak{g}=\mathfrak{h} \oplus \mathfrak{m}$ with $\mathfrak{h}=\operatorname{span}(Y)$ and $\mathfrak{m}=\operatorname{span}\left(Z_{0}, \ldots, Z_{3}\right)$.

One checks that $[\mathfrak{h}, \mathfrak{m}] \subseteq \mathfrak{m}$, i.e. the homogeneous space $G / H$ is reductive. Moreover, if we define

$$
\begin{aligned}
l: \mathfrak{m} & \longrightarrow V_{3} \\
Z_{i} & \longmapsto e_{i}
\end{aligned}
$$

then $\operatorname{ad}(Y)=(l)^{-1} \circ E_{3} \circ l$.

We wish to define an $H_{3}$-connection on $G / H$ such that the canonical action of $G$ on $G / H$ is an action by symmetries. To do this, we need to define a map

$$
\lambda: \mathfrak{m} \rightarrow \mathfrak{h}_{3} \subseteq \mathfrak{g} l\left(V_{3}\right)
$$

such that

$$
\lambda\left(\left[Y, Z_{i}\right]\right)\left[E_{3}, \lambda\left(Z_{i}\right)\right] \quad \text { for } i=0, \ldots, 3
$$

and

$$
\lambda\left(Z_{i}\right) \cdot e_{j}-\lambda\left(Z_{j}\right) \cdot e_{i}=\imath\left(\left[Z_{i}, Z_{j}\right]_{\mathfrak{m}}\right)
$$

with the isomorphism $l$ defined above [KN, X 2.1, 2.3, 4.2]. The covariant derivatives are then defined by $l\left(\nabla_{Z_{i}} Z_{j}\right)=\lambda\left(Z_{i}\right) \cdot e_{j}$, where $T_{p} M$ is identified with $\mathfrak{m}$.

One then checks that the following map satisfies these two conditions and thus defines an $H_{3}$-connection on $M$.

$$
\lambda\left(Z_{0}\right)=0, \quad \lambda\left(Z_{1}\right)=-E_{3}, \quad \lambda\left(Z_{2}\right)=E_{1}, \quad \lambda\left(Z_{3}\right)=3 E_{2} .
$$

The structure polynomials and the structure constant in this case are $a=x^{2}$, $b=\frac{1}{3} x^{3}$, and $c=0$.

Clearly, $G$ acts transitively on the set of parabolas in $\mathbb{R}^{2}$. Also, one sees that $H$ is the group of unimodular affine motions which leave the standard parabola $y=x^{2}$ invariant. Therefore, we can naturally identify $M=G / H$ with the space of parabolas in $\mathbb{R}^{2}$.

Let $\omega_{0}, \ldots, \omega_{3}, \phi$ be the coframe on $G$ dual to the vector fields $Z_{0}, \ldots$, $Z_{3}, Y$. Let $\tau_{i}:=\omega_{i} \wedge \cdots \wedge \omega_{3}$ for $0 \leq i \leq 3 . \tau_{i}$ is invariant under the isotropy representation of $H$, and therefore there are induced forms on $G / H$ which we also denote by $\tau_{0}, \ldots, \tau_{3}$.

One checks that these forms satisfy the Frobenius condition

$$
d \tau_{i}=\alpha_{i} \wedge \tau_{i} \quad \text { for some 1-form } \alpha_{i},
$$

and hence the flag of distributions $\mathscr{D}_{0} \subseteq \cdots \subseteq \mathscr{D}_{3}$ on the tangent space of $G / H$ given by $\tau_{i}\left(\mathscr{D}_{i}\right)=0$ is integrable [EDS, II.1.1].

Let $L: A S l(2, \mathbb{R}) \rightarrow \operatorname{Sl}(2, \mathbb{R})$ be the homomorphism which maps an affine motion to its linear part. Then we can define an angle function

$$
\begin{array}{rlc}
\theta: G / H & \longrightarrow & S^{1} \\
g H & \longmapsto \frac{L(g) \cdot\left(\begin{array}{l}
0 \\
1
\end{array}\right)}{\left\|L(g) \cdot\left(\begin{array}{l}
0 \\
1
\end{array}\right)\right\|} .
\end{array}
$$

This function assigns to each parabola its direction, and it is straightforward to check that $\theta$ is a fibration over $S^{1}$ whose fibers are the maximal connected integral leaves (m.c.i.l.'s) of $\mathscr{D}_{3}$, and that the fibers are totally geodesic. Also, 
since these fibers are all parabolas with a given direction it is easy to see that they are diffeomorphic to $\mathbb{R}^{3}$.

Now let us investigate the level sets of $\theta$. By homogeneity all level sets are equivalent. Thus, let

$$
Y_{3}:=\left\{\left(y=e^{a} x^{2}+b x+c\right) \mid a, b, c \in \mathbb{R}\right\}
$$

Then the function $r_{3}: Y_{3} \rightarrow \mathbb{R}$ which assigns the size to a parabola, i.e. $r_{3}\left(y=e^{a} x^{2}+b x+c\right)=a$, satisfies $d r_{3}=\left.\omega_{2}\right|_{Y_{3}}$. Again, one can check that $r_{3}$ is a fibration of $Y_{3}$ over $\mathbb{R}$ whose fibers are totally geodesic and diffeomorphic to $\mathbb{R}^{2}$.

Next, let $Y_{2}$ be a level set of $r_{3}$, e.g.

$$
Y_{2}:=r_{3}^{-1}(0)=\left\{\left(y=x^{2}+b x+c\right) \mid b, c \in \mathbb{R}\right\}
$$

i.e. a set of parabolas with given direction and size. We compute the connection on $Y_{2}$ and obtain that $Y_{2}$ is flat. Note that a curve $\gamma$ in $Y_{2}$ can be described by the curve of the vertices of $\gamma(t)$. Then we compute that-up to parametrization-the vertices of a geodesic in $Y_{2}$ either move along a parabola of size -2 or along a vertical line.

Also, the restriction $\left.\omega_{1}\right|_{Y_{2}}$ is closed, hence there exists a function $r_{2}: Y_{2} \rightarrow \mathbb{R}$ such that $\left.d r_{2} \omega_{1}\right|_{Y_{2}}$. One finds that the level sets of $r_{2}$ are the parabolas in $Y_{2}$ whose vertices lie on a fixed vertical line. By the previous, these level sets are geodesics.

Finally, if $Y_{1}$ is a level set of $r_{2}$, i.e. a geodesic, we find some parametrization $r_{1}: Y_{1} \rightarrow \mathbb{R}$ such that $d r_{1}=\left.\omega_{0}\right|_{Y_{1}}$.

Therefore, on every m.c.i.l. $Y_{i}$ of $\mathscr{D}_{i}, i \leq 3$, we have a function $r_{i}: Y_{i} \rightarrow \mathbb{R}$ satisfying $d r_{i}=\omega_{i-1} \mid Y_{i}$, and all these functions are totally geodesic fibrations of $Y_{i}$.

2.2 Example II : Types $\Sigma_{0}^{ \pm}$and $\Sigma_{c}^{1}$. Let $c \in \mathbb{R}$ be a given constant and define the Lie algebra $\mathfrak{g}_{c}^{ \pm}=\operatorname{span}\left(Z_{1}, Z_{2}, Z_{3}\right)$ with the bracket relations

$$
\begin{aligned}
& {\left[Z_{1}, Z_{2}\right]=\quad Z_{3},} \\
& {\left[Z_{1}, Z_{3}\right]=-\frac{c}{8} Z_{2},} \\
& {\left[Z_{2}, Z_{3}\right]= \pm 2 c Z_{1} .}
\end{aligned}
$$

One computes that $\mathfrak{g}_{0}^{ \pm} \cong \mathfrak{n}_{3}$, the Lie algebra of the three-dimensional Heisenberg group $N_{3}, \mathfrak{g}_{c}^{+} \cong \mathfrak{s} u(2)$ if $c>0$ and $\mathfrak{g}_{c}^{ \pm} \cong \mathfrak{s l}(2, \mathbb{R})$ in the remaining cases. We let $G_{c}^{ \pm}$be a Lie group corresponding to $\mathfrak{g}_{c}^{ \pm}$such that $G_{0}^{ \pm}=N_{3}$, $G_{c}^{ \pm}=S l(2, \mathbb{R})$ or $G_{c}^{+}=S U(2)$ if $c>0$.

Let $M_{c}^{ \pm}:=\mathbb{R}^{+} \times G_{c}^{ \pm}$and let $t_{0}: M_{c}^{ \pm} \rightarrow \mathbb{R}^{+}$be the projection onto the first factor. Regarding $M_{c}^{ \pm}$as a subset of the Lie group $\mathbb{R} \times G_{c}^{ \pm}$we can define left invariant vector fields $Z_{0}, \ldots, Z_{3}$ such that

for $1 \leq i, j \leq 3,\left[Z_{i}, Z_{j}\right]$ is given by the equations of $\mathfrak{g}_{c}^{ \pm}$, $\left[Z_{0}, Z_{i}\right]=0$ for $i=1,2,3$,

$$
Z_{0}\left(t_{0}\right) \equiv 1 \text {, and } Z_{i}\left(t_{0}\right) \equiv 0, i=1,2,3 \text {. }
$$


We then define a frame on $M_{c}^{ \pm}$by

$$
\begin{array}{llll}
X_{0} & = & -\frac{\left(6 t_{0}^{2} \mp c\right)}{t_{0}} Z_{1} & -4 Z_{3} \\
X_{1} & =\frac{3}{2}\left(2 t_{0}^{2} \pm c\right) Z_{0} & \multicolumn{2}{c}{-2 Z_{2}} \\
X_{2} & \mp \quad \frac{2}{t_{0}} Z_{1} \\
X_{3} & \mp 3 Z_{0}
\end{array}
$$

Now we give a connection w.r.t. this frame by the $\mathfrak{h}_{3}$-valued 1 -form

$$
\phi_{c}^{ \pm}=\phi_{1} E_{1}+\phi_{2} E_{2}+\phi_{3} E_{3}
$$

where

$$
\begin{array}{ll}
\phi_{1}= & -2 t_{0} \omega_{1}, \\
\phi_{2}=c \frac{2 t_{0}^{2} \pm c}{4 t_{0}} \omega_{0} & -\frac{4 t_{0}^{2} \pm c}{2 t_{0}} \omega_{2}, \quad \text { and } \\
\phi_{3}=-\frac{6 t_{0}^{2} \pm c}{2 t_{0}} \omega_{0} & \pm \quad \frac{1}{t_{0}} \omega_{2} .
\end{array}
$$

The covariant derivative is defined by (3). Moreover, one checks that (4) is satisfied, i.e. $\nabla$ is torsion free. Also, $\nabla$ is not flat, thus by Corollary 3.2 we conclude that $\nabla$ is an $H_{3}$-connection.

The natural left action of $G_{c}^{ \pm}$on $M_{c}^{ \pm}$is an action by symmetries as it leaves all $Z_{i}$ and the function $t_{0}$ invariant, hence preserves the frame $X_{0}, \ldots X_{3}$ and therefore satisfies condition (1).

The structure polynomials are

$$
a= \pm x^{2}+\frac{1}{2}\left(2 t_{0}^{2} \pm c\right) y^{2}, \quad \text { and } \quad b=t_{0} y\left(\mp x^{2}+\frac{2 t_{0}^{2} \pm c}{6} y^{2}\right) .
$$

We shall see later that $M_{c_{1}}^{\varepsilon_{1}}$ and $M_{c_{2}}^{\varepsilon_{2}}$ with $\varepsilon_{i} \in\{ \pm\}$ are equivalent iff $\varepsilon_{1}=$ $\varepsilon_{2}$ and $\operatorname{sign}\left(c_{1}\right) \operatorname{sign}\left(c_{2}\right)$. Thus the $M_{c}^{ \pm}$give six different examples of $H_{3^{-}}$ connections.

2.3 Example III : Type $\Sigma_{c}^{2}$. Let $k \in \mathbb{R} \backslash\{0\}$ be a given constant and define the Lie algebra $\mathfrak{g}_{k}^{ \pm}=\operatorname{span}\left(Z_{1}, Z_{2}, Z_{3}\right)$ with the bracket relations

$$
\begin{aligned}
& {\left[Z_{1}, Z_{2}\right]=Z_{3},} \\
& {\left[Z_{1}, Z_{3}\right]=\mp Z_{2},} \\
& {\left[Z_{2}, Z_{3}\right]=\mp 3 k Z_{1} .}
\end{aligned}
$$

One computes that $\mathfrak{g}_{k}^{+} \cong \mathfrak{s} u(2)$ if $k>0$ and $\mathfrak{g}_{k}^{ \pm} \cong \mathfrak{s l}(2, \mathbb{R})$ in the remaining cases. We let $G_{k}^{ \pm}$be a Lie group corresponding to $\mathfrak{g}_{k}^{ \pm}$such that $G_{k}^{ \pm}=\operatorname{Sl}(2, \mathbb{R})$ or $G_{k}^{+}=S U(2)$.

Let $M_{k}^{ \pm}:=\mathbb{R}^{+} \times G_{k}^{ \pm}$and let $t_{0}: M_{k}^{ \pm} \rightarrow \mathbb{R}^{+}$be the projection onto the first factor. Define the vector fields $Z_{0}, \ldots Z_{3}$ on $M_{k}^{ \pm}$as in the previous section, i.e. the relations of $\mathfrak{g}_{k}^{ \pm}$determine $\left[Z_{i}, Z_{j}\right]$ for $1 \leq i, j \leq 3, Z_{0}\left(t_{0}\right) \equiv 1$, $Z_{i}\left(t_{0}\right) \equiv 0$, and $\left[Z_{0}, Z_{i}\right]=0$ for $i=1,2,3$. 
We then define a frame on $M_{k}^{ \pm}$by

$$
\begin{aligned}
X_{0} & = & \frac{9}{t_{0}}\left(k t_{0}^{2} \pm 1\right) & Z_{1} \\
X_{1} & =\left(3 k t_{0}^{2} \mp 1\right) Z_{0} & & \\
X_{2} & = & \frac{3}{t_{0}} Z_{1}, & \\
X_{3} & = & -3 Z_{0} . &
\end{aligned}
$$

Now we give a connection w.r.t. this frame by the $\mathfrak{h}_{3}$-valued 1 -form

$$
\phi_{k}^{ \pm}=\phi_{1} E_{1}+\phi_{2} E_{2}+\phi_{3} E_{3}
$$

where

$$
\begin{aligned}
& \phi_{1}=-2 k t_{0} \omega_{1}, \\
& \phi_{2}=\frac{3}{t_{0}}\left( \pm 3 k t_{0}^{2}-1\right) \omega_{0} \quad-\frac{1}{t_{0}}\left(2 k t_{0}^{2} \pm 1\right) \omega_{2}, \quad \text { and } \\
& \phi_{3}=-\frac{3}{t_{0}}\left(k t_{0}^{2} \mp 1\right) \omega_{0}+t^{2} \quad \frac{1}{t_{0}} \omega_{2} .
\end{aligned}
$$

Again, the covariant derivative is defined by (3). Moreover, one checks that (4) is satisfied, i.e. $\nabla$ is torsion free. Also, $\nabla$ is not flat for $k \neq 0$, thus by Corollary 3.2 we conclude that $\nabla$ is an $H_{3}$-connection.

The natural left action of $G_{k}^{ \pm}$on $M_{k}^{ \pm}$is an action by symmetries for similar reasons as in the previous section.

The structure polynomials and the structure constant are

$$
a\left(k t_{0} y\right)^{2}+k\left(x^{2} \pm y^{2}\right), b=\frac{1}{3} k t_{0} y\left(\left(k t_{0} y\right)^{2}-3 k\left(x^{2} \pm y^{2}\right)\right) \quad \text { and } \quad c= \pm 6 k^{2} \text {. }
$$

We shall see later that $M_{k_{1}}^{\varepsilon_{1}}$ and $M_{k_{2}}^{\varepsilon_{2}}$ with $\varepsilon_{i} \in\{ \pm\}$ are equivalent iff $\varepsilon_{1}=\varepsilon_{2}$ and $\operatorname{sign}\left(k_{1}\right)=\operatorname{sign}\left(k_{2}\right)$. Thus the $M_{k}^{ \pm}$give four different examples of $\boldsymbol{H}_{3}$ connections, and we will also show that they are indeed different from the connections given in the previous section.

\section{The structure equations}

In this section we will mainly recall the results of Bryant [Br2] on $\mathrm{H}_{3}$ connections and introduce a notation convenient for our purposes.

First, we shall define the bilinear parings

$$
\langle,\rangle_{p}: V_{n} \otimes V_{m} \rightarrow V_{n+m-2 p}
$$

by

$$
\langle u, v\rangle_{p}=\frac{1}{p !} \sum_{k=0}^{p}(-1)^{k}\left(\begin{array}{l}
p \\
k
\end{array}\right) \frac{\partial^{p} u}{\partial^{k} x \partial^{p-k} y} \frac{\partial^{p} v}{\partial^{p-k} x \partial^{k} y} \quad \text { for } u \in V_{n}, v \in V_{m}
$$

It can be shown that these pairing are $S l(2, \mathbb{R})$-equivariant and therefore are the projections onto the summands of the Clebsch-Gordan formula.

We can establish an $S l(2, \mathbb{R})$-equivariant isomorphism $\rho: \mathfrak{h}_{3} \rightarrow V_{2}$ by the equation

$$
A \cdot v=\langle\rho(A), v\rangle_{1} \quad \text { with } A \in \mathfrak{h}_{3}, v \in V_{3},
$$

and we will from now on use $\rho$ to identify $\mathfrak{h}_{3}$ and $V_{2}$. 
Now we describe the spaces $\mathbf{K}\left(\mathfrak{h}_{3}\right)$ and $\mathbf{K}^{1}\left(\mathfrak{h}_{3}\right)$ defined in $\S 1$. Straightforward calculation yields that we can define $\operatorname{Sl}(2, \mathbb{R})$-equivariant isomorphisms

$$
\imath: \mathbf{K}\left(\mathfrak{h}_{3}\right) \rightarrow V_{2} \quad \text { and } \quad l_{1}: \mathbf{K}^{1}\left(\mathfrak{h}_{3}\right) \rightarrow V_{3}
$$

by the equations

$$
\varphi(u, v)=\left\langle l(\varphi),\langle u, v\rangle_{3}\right\rangle_{0}-\frac{1}{12}\left\langle l(\varphi),\langle u, v\rangle_{1}\right\rangle_{2}
$$

and

$$
\imath(\psi(u))=\left\langle\iota_{1}(\psi), u\right\rangle_{2}
$$

where $\varphi \in \mathbf{K}\left(\mathfrak{h}_{3}\right), \psi \in \mathbf{K}^{1}\left(\mathfrak{h}_{3}\right)$ and $u, v \in V_{3}$, and we will from now on use these to identify $\mathbf{K}$ and $\mathbf{K}^{1}\left(\mathfrak{h}_{3}\right)$ with $V_{2}$ and $V_{3}$ resp.

The following lemma is very simple but will be useful later on.

Lemma 3.1. Every $\varphi \in \mathbf{K}\left(\mathfrak{h}_{3}\right) \backslash 0$ is surjective.

Proof. Let $\varphi=a_{0} x^{2}+a_{1} x y+a_{2} y^{2} \in V_{2} \cong \mathbf{K}\left(\mathfrak{h}_{3}\right)$, and suppose $a_{0} \neq 0$. Then $\varphi\left(e_{2}, e_{3}\right)=-36 a_{0} y^{2}$, thus $y^{2} \in \operatorname{im}(\varphi)$. Also, $\varphi\left(e_{1}, e_{3}\right)=-36 a_{0} x y+18 a_{1} y^{2}$, thus $x y \in \operatorname{im}(\varphi)$. Finally, $\varphi\left(e_{0}, e_{3}\right)=-18 a_{0} x^{2}+36 a_{1} x y-18 a_{2} y^{2}$, thus $x^{2} \in \operatorname{im}(\varphi)$. Therefore, $\varphi$ is surjective if $a_{0} \neq 0$. Similar arguments show that $\varphi$ is surjective whenever $\varphi \neq 0$.

Corollary 3.2. If the holonomy group of a torsion free connection $\nabla$ on a manifold $M$ is contained in $\mathrm{H}_{3}$ then either the holonomy group of $\nabla$ is equal to $\mathrm{H}_{3}$ or $\nabla$ is flat.

Proof. Suppose $\nabla$ is not flat. Then there is a $p \in M$ at which the curvature map $\Omega_{p}: \Lambda^{2} T_{p} M \rightarrow h_{3}$ is not 0 . By Lemma 3.1, $\Omega_{p}$ is surjective and thus by the Ambrose-Singer-Holonomy Theorem, the holonomy group of $\nabla$ is equal to $\mathrm{H}_{3}$.

Suppose $M$ is equipped with a torsion free $H_{3}$-connection $\nabla$. Let $\pi: \mathfrak{F} \rightarrow$ $M$ denote the total coframe bundle of $M$ which is a principal $G l(4, \mathbb{R})$-bundle. On $\mathfrak{F}$, the tautological 1-form $\omega$ takes values in $\mathbb{R}^{4}$, the connection 1-form $\phi$ takes values in $\mathfrak{g} l(4, \mathbb{R})$ and both are $G l(4, \mathbb{R})$-equivariant $[\mathrm{KN}]$.

Then there exists a reduction $F$ of $\mathfrak{F}$ whose structure group is isomorphic to $\operatorname{Sl}(2, \mathbb{R})[\mathrm{KN}, \mathrm{II} .7 .1]$ and such that $\left.\phi\right|_{F}$ takes values in $\underline{\mathfrak{h}}_{3} \subseteq \mathfrak{g l}(4, \mathbb{R})$. Such a reduction will be called an $\mathfrak{h}_{3}$-reduction of $\mathfrak{F}$.

By abuse of notation we will denote the restrictions $\left.\omega\right|_{F},\left.\phi\right|_{F}$ and $\left.\pi\right|_{F}$ also by $\omega, \phi$ and $\pi$ resp.

Of course, $\mathfrak{h}_{3}$-reductions are not unique. In fact, one sees that $F, F^{\prime}$ are $\mathfrak{h}_{3}$-reductions iff there is some $g \in \operatorname{Norm}\left(\underline{h}_{3}\right) \subseteq G l(4, \mathbb{R})$ with $F^{\prime}=L_{g}(F)$, where $L_{g}$ denotes the principal action of $g$ on $\mathfrak{F}$. Now one computes that

$$
\operatorname{Norm}\left(\underline{\mathfrak{h}}_{3}\right)=H_{3} \times N
$$

with

$$
N=\left\{\left(\begin{array}{cccc}
t & & & \\
& \varepsilon t & & \\
& & t & \\
& & & \varepsilon t
\end{array}\right) \mid t \in \mathbb{R} \backslash\{0\}, \varepsilon= \pm 1\right\} .
$$


It follows that any two $\mathfrak{h}_{3}$-reductions $F, F^{\prime}$ satisfy $F=L_{g}\left(F^{\prime}\right)$ for some $g \in N$, and two such structures are called homothetic to each other.

Clearly, homothety is an equivalence relation on the set of $\mathfrak{h}_{3}$-reductions of $\mathfrak{F}$, and it is not hard to see that there is in fact a 1-1 correspondence between $H_{3}$-connections and homothety classes of $\mathfrak{h}_{3}$-reductions with a torsion free connection [Br2, 2.1].

Let $M$ be as above and fix an $\mathfrak{h}_{3}$-reduction $F$ of the total coframe bundle $\mathfrak{F}$. On $F$, we have the 1-form $\omega+\phi$ with values in $\mathbb{R}^{4} \oplus \underline{\mathfrak{h}}_{3} \cong V_{3} \oplus V_{2}$ and may hence regard $\omega+\phi$ as an $\operatorname{Sl}(2, \mathbb{R})$-equivariant $V_{3} \oplus V_{2}$-valued 1-form. It is well known that this form gives a coframe on $F$, i.e. the real-valued 1-forms $\omega_{0}, \ldots, \omega_{3}, \phi_{0}, \phi_{1}, \phi_{2}$ given by

$$
\omega(X)=\sum_{i} \omega_{i}(X) e_{i} \quad \text { and } \quad \phi(X)=\sum_{i} \phi_{i}(X) x^{2-i} y^{i} \quad \text { for all } \quad X \in T F
$$

are linearly independent.

The frame of $F$ dual to the coframe $\omega_{0}, \ldots, \omega_{3}, \phi_{0}, \phi_{1}, \phi_{2}$ will be denoted by $X_{0}, \ldots, X_{3}, Y_{0}, Y_{1}, Y_{2}$ and will be called the canonical frame on $F$.

A tangent vector $X \in T F$ will be called vertical if $\omega(X)=0$ and horizontal if $\phi(X)=0$. Thus, $X_{i}$ is horizontal and $Y_{i}$ is vertical for all $i$.

The curvature 2 -form $\Omega$ on $F$ takes values in $\mathfrak{h}_{3} \cong V_{2}$ and vanishes in vertical directions which means that for every $u \in F$, there is a unique linear map $\varphi_{u}: \Lambda^{2} V_{3} \rightarrow \mathfrak{h}_{3}$ such that

$$
\Omega(X, Y)=\varphi_{u}(\omega(X), \omega(Y)) \quad \text { for all } X, Y \in T_{u} F \text {. }
$$

Furthermore, the first Bianchi identity implies that $\varphi_{u} \in \mathbf{K}\left(\mathfrak{h}_{3}\right)$, and this defines an $S l(2, \mathbb{R})$-equivariant map

$$
\begin{aligned}
a: F & \longrightarrow V_{2} \\
u & \longmapsto \varphi_{u} .
\end{aligned}
$$

For $u \in F$, we define a linear map $\psi_{u}: V_{3} \rightarrow \operatorname{Hom}\left(\Lambda^{2} V_{3}, \mathfrak{h}_{3}\right)$ by the equation

$$
\begin{aligned}
\psi_{u}\left(e_{i}\right)(\omega(Y), \omega(Z))= & \left(\mathfrak{L}_{X_{i}} \Omega\right)(Y, Z)-\varphi_{u}\left(\phi(Y) \cdot e_{i}, \omega(Z)\right) \\
& -\varphi_{u}\left(\omega(Y), \phi(Z) \cdot e_{i}\right) \text { for all } Y, Z \in T_{u} F .
\end{aligned}
$$

To see that this is well defined note that the right-hand side is tensorial in $Y$ and $Z$ and vanishes if either $Y$ or $Z$ are vertical. Moreover, the first Bianchi identity implies that $\psi_{u}\left(e_{i}\right) \in \mathbf{K}\left(\mathfrak{h}_{3}\right)$, and finally, the second Bianchi identity implies that $\psi_{u} \in \mathbf{K}^{1}\left(\mathfrak{h}_{3}\right) \cong V_{3}$. Therefore, we get a map

$$
\begin{aligned}
b: F & \longrightarrow V_{3} \\
u & \longmapsto \psi_{u} .
\end{aligned}
$$

Now we can describe the structure equations on $F$.

The first structure equation-using that $\nabla$ is torsion free-yields

$$
d \omega=-\phi \wedge \omega=-\langle\phi, \omega\rangle_{1} .
$$

The second structure equation is

$$
\begin{aligned}
d \phi & =-\phi \wedge \phi+\Omega \\
& =-\frac{1}{2}\langle\phi, \phi\rangle_{1}+\left\langle a,\langle\omega, \omega\rangle_{3}\right\rangle_{0}-\frac{1}{12}\left\langle a,\langle\omega, \omega\rangle_{1}\right\rangle_{2} .
\end{aligned}
$$


Next, we compute the exterior derivatives of $a$. For this, let $X_{i}, X_{j}$ and $X_{k}$ be horizontal vector fields of the canonical frame on $F$. Then, at some point $u \in F$,

$$
\begin{aligned}
X_{i}\left(\Omega\left(X_{j}, X_{k}\right)\right) & =\left(\mathfrak{L}_{X_{i}} \Omega\right)\left(X_{j}, X_{k}\right)+\Omega\left(\left[X_{i}, X_{j}\right], X_{k}\right)+\Omega\left(X_{j},\left[X_{i}, X_{k}\right]\right) \\
& =\psi_{u}\left(e_{i}\right)\left(e_{j}, e_{k}\right) \\
& =\left\langle\left\langle b(u), e_{i}\right\rangle_{2},\left\langle e_{j}, e_{k}\right\rangle_{3}\right\rangle_{0}-\frac{1}{12}\left\langle\left\langle b(u), e_{i}\right\rangle_{2},\left\langle e_{j}, e_{k}\right\rangle_{1}\right\rangle_{2} .
\end{aligned}
$$

Here we used the fact that the Lie bracket of two canonical horizontal vector fields is always vertical since the connection is torsion free.

On the other hand,

$$
X_{i}\left(\Omega\left(X_{j}, X_{k}\right)\right)=\left\langle\left\langle X_{i}(a), e_{i}\right\rangle_{2},\left\langle e_{j}, e_{k}\right\rangle_{3}\right\rangle_{0}-\frac{1}{12}\left\langle X_{i}(a),\left\langle e_{j}, e_{k}\right\rangle_{1}\right\rangle_{2} .
$$

Setting these two equal, we get the horizontal derivatives of $a$. The vertical derivatives follow from the $\operatorname{Sl}(2, \mathbb{R})$-equivariance of $a$, and we obtain

$$
d a=\langle b, \omega\rangle_{2}+\langle a, \phi\rangle_{1} .
$$

Taking the exterior derivative of this equation and solving for $d b$, we can compute that there exists some function $c$ such that

$$
d b=\langle b, \phi\rangle_{1}+\left(c-\langle a, a\rangle_{2}\right) \omega+\frac{1}{12}\left\langle\langle a, a\rangle_{0}, \omega\right\rangle_{2} .
$$

Taking exterior derivatives once again, we find that

$$
d c=0 \text {, }
$$

i.e. $c$ is a constant.

Definition 3.3. Let $\pi: F \rightarrow M$ be a principal $S l(2, \mathbb{R})$-bundle over a fourdimensional manifold $M$. Suppose there exist 1-forms $\omega$ and $\phi$ on $F$ with values in $V_{3}$ and $\mathfrak{h}_{3}$ resp. and functions $a$ and $b$ with values in $V_{2}, V_{3}$ resp. such that the structure equations (5)-(9) are satisfied for some constant $c$. Moreover, assume that $\omega\left(\operatorname{ker}\left(\pi_{*}\right)\right) \equiv 0$ and $\phi\left(\left(\tilde{E}_{i}\right)^{*}\right)=E_{i}$ where $\left\{\tilde{E}_{i}\right\}$ is the basis of $\mathfrak{s l}(2, \mathbb{R})$ described earlier and $\left(\tilde{E}_{i}\right)^{*}$ denotes the fundamental vector field corresponding to $\tilde{E}_{i}[\mathrm{KN}, \mathrm{Vol} \mathrm{I}, \mathrm{p}$. 51]. Then $(\pi, F, M, \omega, \phi, a, b, c)$ is called a solution structure over $M$.

By the previous discussion we know that any $\mathfrak{h}_{3}$-reduction of an $\mathrm{H}_{3}$-connection on $M$ gives rise to a solution structure over $M$.

Now suppose that $F^{\prime}=L_{g}(F)$ with $g \in N$ is an $\mathfrak{h}_{3}$-reduction homothetic to $F$. Then if we let $\tilde{a}=L_{g} \circ a, \tilde{b}=L_{g} \circ b$ and $\tilde{c}=L_{g} \circ c$ where $L_{g}$ denotes the action of $g$ on $V_{2}, V_{3}$ resp., then $\left(F^{\prime},\left(L_{g^{-1}}\right)^{*}(\omega),\left(L_{g^{-1}}\right)^{*}(\phi), \tilde{a}, \tilde{b}, \tilde{c}\right)$ is also a solution structure.

If we define the components of the polynomials $a$ and $b$ by $a=\sum_{i} a_{i} x^{2-i} y^{i}$ and $b=\sum_{i} b_{i} x^{3-i} y^{i}$ then we compute that if

$$
g=\left(\begin{array}{llll}
t & & & \\
& \varepsilon t & & \\
& & t & \\
& & & \varepsilon t
\end{array}\right) \in N
$$


then

$$
\begin{aligned}
& \tilde{a} t^{2}=\left(a_{0} x^{2}+\varepsilon a_{1} x y+a_{2} y^{2}\right), \\
& \tilde{b}=t^{3}\left(\varepsilon b_{0} y^{3}+b_{1} x^{2} y+\varepsilon b_{2} x y^{2}+b_{3} y^{3}\right), \\
& \tilde{c}=t^{4} c .
\end{aligned}
$$

Definition 3.4. Two solution structures $(\pi, F, M, \omega, \phi, a, b, c)$ and $(\tilde{\pi}, \tilde{F}$, $M, \tilde{\omega}, \tilde{\phi}, \tilde{a}, \tilde{b}, \tilde{c})$ over the same manifold $M$ are called homothetic if there exists a bundle isomorphism $L: F \rightarrow \tilde{F}$ such that $\omega=L^{*}(\tilde{\omega}), \phi=L^{*}(\tilde{\phi})$, and moreover $(a, b, c)$ and $(\tilde{a}, \tilde{b}, \tilde{c})$ satisfy (10) for some $t \neq 0, \varepsilon= \pm 1$.

Clearly, homothety is an equivalence relation of solution structures and homothetic $\mathfrak{h}_{3}$-reductions give rise to homothetic solution structures. Therefore, by our discussion earlier, to any $H_{3}$-connection on a manifold $M$ we can associate a homothety class of solution structures over $M$.

We will now show that this correspondence is in fact bijective. In particular, we need to show the sufficiency of the structure equations (5)-(9).

Proposition 3.5. Let $M$ be a 4-dimensional manifold and let $\pi: \mathfrak{F} \rightarrow M$ denote the total coframe bundle of $M$. Suppose there exists a solution structure $(\bar{\pi}, \bar{F}, M, \bar{\omega}, \bar{\phi}, \bar{a}, \bar{b}, c)$ over $M$.

Then there exists a unique $H_{3}$-connection $\nabla$ on $M$ and a unique embedding $\imath: \bar{F} \hookrightarrow \mathfrak{F}$ such that

(1) $F:=\imath(\bar{F})$ is an $\mathfrak{h}_{3}$-reduction of $\mathfrak{F}$,

(2) the diagram

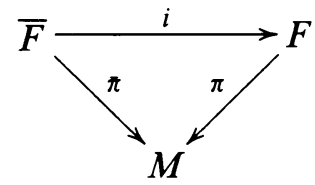

commutes and

(3) $l^{*}(\omega+\phi)=\bar{\omega}+\bar{\phi}$, where $\omega$ and $\phi$ denote the restrictions of the tautological form and the connection form to $F$.

Proof. Let $\bar{F}, \bar{\omega}, \bar{\phi}, \bar{a}$ and $\bar{b}$ be as above. By hypothesis, $\lambda \circ \bar{\omega}$ with the identification $\lambda: V_{3} \rightarrow \mathbb{R}^{4}$ as before (§2) is a coframe of $T_{\pi(u)} M$ for all $u \in \bar{F}$, hence a point in $\mathfrak{F}$. Therefore, we define the map

$$
\begin{aligned}
l: \bar{F} & \longrightarrow \mathfrak{F} \\
u & \longmapsto \lambda \circ \bar{\omega}
\end{aligned}
$$

By construction, we have $l^{*}(\omega)=\bar{\omega}$. Moreover, the structure equations and the condition $\phi\left(\left(\tilde{E}_{i}\right)^{*}\right)=E_{i}$ imply that $\bar{\omega}$ and $\bar{\phi}$ are $S l(2, \mathbb{R})$-equivariant w.r.t. the actions of $S l(2, \mathbb{R})$ on $V_{3}$ and $\mathfrak{h}_{3}$ induced by $\rho_{3}$. This means that

- $l$ is an embedding whose image $F$ is an $\mathfrak{h}_{3}$-reduction of $\mathfrak{F}$ w.r.t. the connection defined by $\phi$.

- if we define on $F:=\imath(\bar{F})$ the $\mathfrak{h}_{3}$-valued 1 -form $\phi^{\prime}:=\left(l^{-1}\right)^{*}(\bar{\phi})$ then there exists a unique $\mathfrak{g l}(4, \mathbb{R})$-valued $G l(4, \mathbb{R})$-equivariant connection 1 -form $\phi$ on $\mathfrak{F}$ such that $\phi^{\prime}=\left.\phi\right|_{F}$,

It is then straightforward that the map $l$ satisfies the desired properties and is uniquely determined by them. 
From the construction above it is clear that the images of the embeddings $l_{1}$ and $l_{2}$ associated to two homothetic solution structures $\left(\bar{\pi}_{i}, \bar{F}_{i}, M, \bar{\omega}_{i}, \bar{\phi}_{i}\right.$, $\left.\bar{a}_{i}, \bar{b}_{i}, c_{i}\right)$ over $M$ are in fact homothetic $\mathfrak{h}_{3}$-structures. In particular, the connection $\nabla$ on $M$ only depends on the homothety class of the solution structure.

We summarize the discussion so far, including Corollary 3.2, in the

Corollary 3.6. There is a 1-1 correspondence between $H_{3}$-connections on a 4manifold $M$ and homothety classes of solution structures over $M$ for which $a, b$ do not vanish identically.

For the rest of this section, let $F$ be an $\mathfrak{h}_{3}$-reduction of an $H_{3}$-connection $\nabla$ on a connected 4-manifold $M$. If we let $V:=V_{2} \oplus V_{3}$, we get a map

$$
\begin{array}{ccc}
K: \quad F & \longrightarrow & V \\
u & \longmapsto a(u)+b(u)
\end{array}
$$

The structure equations (5)-(9) imply that w.r.t. the canonical frame $X_{0}, \ldots$, $X_{3}, Y_{0}, Y_{1}, Y_{2}$ on $F$ and the components $a_{0}, a_{1}, a_{2}$ and $b_{0}, \ldots, b_{3}$ of the polynomials $a$ and $b$ resp. we can write the differential of $K$

$$
K_{*}: T F \rightarrow T V
$$

as

$$
K_{*}=\left(\begin{array}{ccccccc}
6 b_{2} & -4 b_{1} & 6 b_{0} & 0 & 2 a_{1} & -2 a_{0} & 0 \\
18 b_{3} & -2 b_{2} & -2 b_{1} & 18 b_{0} & 4 a_{2} & 0 & -4 a_{0} \\
0 & 6 b_{3} & -4 b_{2} & 6 b_{1} & 0 & 2 a_{2} & -2 a_{1} \\
p_{1} & -a_{0} a_{1} & a_{0}^{2} & 0 & 2 b_{1} & -3 b_{0} & 0 \\
3 a_{1} a_{2} & p_{2} & 0 & 3 a_{0}^{2} & 4 b_{2} & -b_{1} & -6 b_{0} \\
3 a_{2}^{2} & 0 & p_{2} & 3 a_{0} a_{1} & 6 b_{3} & b_{2} & -4 b_{1} \\
0 & a_{2}^{2} & -a_{1} a_{2} & p_{1} & 0 & 3 b_{3} & -2 b_{2}
\end{array}\right),
$$

where $p_{1}=c+\frac{3}{2} a_{1}^{2}-3 a_{0} a_{2}$ and $p_{2}=c+\frac{1}{2} a_{1}^{2}-5 a_{0} a_{2}$.

We compute that $\operatorname{det}\left(K_{*}\right) \equiv 0$, i.e. $\operatorname{rank}\left(K_{*}\right) \leq 6$. Let $L$ be the cofactor matrix of $K_{*}$, consisting of the $6 \times 6$ minors of $K_{*}$. L has the property that $L K_{*}=K_{*} L=0$, and $L$ has rank at most 1 . Thus, there are polynomials $r_{0}, \ldots, r_{6}, s_{0}, \ldots, s_{6}$ in the variables $a_{0}, a_{1}, a_{2}, b_{0}, \ldots, b_{3}$ such that

$$
L=\left(\begin{array}{c}
r_{0} \\
\vdots \\
r_{6}
\end{array}\right)\left(s_{0}, \ldots, s_{6}\right) .
$$

Furthermore, if we define the polynomial

$$
R_{c}=\frac{1}{4}\left\langle p_{c}, p_{c}\right\rangle_{2}+\frac{1}{2}\left\langle a, q^{2}\right\rangle_{2}
$$

with

$$
p_{c}=\left(2 c-\langle a, a\rangle_{2}\right) a-\langle b, b\rangle_{2} \quad \text { and } \quad q=\langle a, b\rangle_{2},
$$

then it turns out that

$$
s_{i}=\frac{\partial R_{c}}{\partial a_{i}} \quad \text { for } \quad 0 \leq i \leq 2, \quad \text { and } \quad s_{i}=\frac{\partial R_{c}}{\partial b_{i-3}} \quad \text { for } \quad 3 \leq i \leq 6,
$$

and

$$
r_{i}=k_{i} s_{6-i} \quad \text { where }\left(k_{0}, \ldots, k_{6}\right)=\frac{1}{6}(1,-3,3,-1,3,-6,3) .
$$


This means that

- $d\left(R_{c} \circ K\right) \equiv 0$, hence $K$ maps $F$ into some level set of $R_{c}$,

- for $u \in F, \operatorname{rank}\left(K_{*}(u)\right)=6$ iff $L(u) \neq 0$ iff $\left(r_{0}(u), \ldots, r_{6}(u)\right) \neq 0$ iff $\left(s_{0}(u), \ldots, s_{6}(u)\right) \neq 0$ iff $K(u)$ is a regular point of $R_{c}$.

We now turn our attention to the set $\Sigma_{c} \subseteq V$ of critical points of $R_{c}$. Using the $\operatorname{Sl}(2, \mathbb{R})$-invariance of $\Sigma_{c}$, we compute that

- for $c \neq 0, \Sigma_{c}=\Sigma_{c}^{1} \sqcup \Sigma_{c}^{2}$, where

$$
\Sigma_{c}^{1}=\left\{(a, b) \in V \mid p_{c}=q=0\right\}
$$

and

$\Sigma_{c}^{2}=\left\{\left(v+u^{2}, \frac{1}{3} u\left(u^{2}-3 v\right)\right) \in V \mid u \in V_{1}, v \in V_{2}\right.$ with $\left.\langle v, v\rangle_{2}=\frac{2}{3} c\right\}$.

- for $c=0, \Sigma_{0}=\Sigma_{0}^{+} \cup \Sigma_{0}^{-}$, where

$$
\Sigma_{0}^{ \pm}=\left\{\left( \pm v^{2}+u^{2}, \frac{1}{3} u\left(\mp 3 v^{2}+u^{2}\right)\right) \in V \mid u, v \in V_{1}\right\}
$$

We also define

$$
\Sigma_{0}^{0}:=\Sigma_{0}^{+} \cap \Sigma_{0}^{-}=\left\{\left(u^{2}, \frac{1}{3} u^{3}\right) \in V \mid u \in V_{1}\right\} .
$$

We wish to determine the topology of the $\Sigma$ 's. Of special interest for us is the number of connected components in each case. To determine this we introduce the $S l(2, \mathbb{R})$-equivariant maps

$$
\begin{array}{cccc}
\varphi_{c}^{2}: & V_{1} \times V_{2, c} & \longrightarrow & \Sigma_{c}^{2} \\
(u, v) & \longmapsto & \left(v+u^{2}, \frac{1}{3} u\left(u^{2}-3 v\right)\right), \\
& & & \Sigma_{0}^{ \pm} \\
\varphi_{0}^{ \pm}: & V_{1} \times V_{1} & \longrightarrow & \\
& (u, v) & \longmapsto & \left( \pm v^{2}+u^{2}, \frac{1}{3} u\left(\mp 3 v^{2}+u^{2}\right)\right), \\
\varphi_{0}^{0}: & V_{1} & \longrightarrow & \Sigma_{0}^{0} \\
& u & \longmapsto & \left(u^{2}, \frac{1}{3} u^{3}\right),
\end{array}
$$

where $V_{2, c}:=\left\{v \in V_{2} \mid\langle v, v\rangle_{2}=\frac{2}{3} c\right\}$.

One computes that $\varphi_{c}^{2}$ is a diffeomorphism for all $c$. It follows that $\Sigma_{c}^{2}$ is smooth 4-dimensional and has one or two connected components depending on the sign of $c$. In the case $c>0$ we let $V_{2, c}^{ \pm}=V_{2, c} \cap\left\{ \pm\left(u^{2}+v^{2}\right) \mid u, v \in V_{1}\right\}$ be the connected components of $V_{2, c}$, and therefore $\Sigma_{c}^{2, \pm}:=\varphi_{c}^{2}\left(V_{1} \times V_{2, c}^{ \pm}\right)$are the two connected components of $\Sigma_{c}^{2}$. On the other hand, if $c<0$ then $V_{2, c}$ and therefore $\Sigma_{c}^{2}$ are connected.

$\varphi_{0}^{ \pm}$is a branched double cover: in fact, let

$$
\begin{aligned}
& S^{+}:=\left(\varphi_{0}^{+}\right)^{-1}\left(\Sigma_{0}^{0}\right)=\left\{(u, v) \in V_{1} \times V_{1} \mid v=0 \text { or } v^{2}=3 u^{2}\right\}, \text { and } \\
& S^{-}:=\left(\varphi_{0}^{-}\right)^{-1}\left(\Sigma_{0}^{0}\right)=\left\{(u, v) \in V_{1} \times V_{1} \mid v=0\right\} .
\end{aligned}
$$

Then $\left.\varphi_{0}^{ \pm}\right|_{\left(V_{1} \times V_{1}\right) \backslash S^{ \pm}}$is a double cover of $\Sigma_{0}^{ \pm} \backslash \Sigma_{0}^{0}$ whose nontrivial deck transformation in either case is given by $(u, v) \mapsto(u,-v)$.

It follows that $\Sigma_{0} \backslash \Sigma_{0}^{0}$ is 4-dimensional and smooth and has two connected components, namely $\Sigma_{0}^{ \pm} \backslash \Sigma_{0}^{0}$.

Next, $\left.\varphi_{0}^{0}\right|_{V_{1} \backslash\{0\}}$ is a diffeomorphism onto $\Sigma_{0}^{0} \backslash\{0\}$, thus $\Sigma_{0}^{0} \backslash\{0\}$ is a smooth connected surface. 
Note that $\Sigma_{c}^{1}$ is smooth by the implicit function theorem. Let $(a, b) \in$ $\Sigma_{c}^{1}$ with $\langle a, a\rangle_{2}>0$. By the $S l(2, \mathbb{R})$-invariance we may assume that $a=$ $a_{0}\left(x^{2}+y^{2}\right)$ for some $a_{0} \in \mathbb{R} \backslash\{0\}$. We compute that $b=b_{1} y\left(3 x^{2}-y^{2}\right)+$ $b_{2} x\left(3 y^{2}-x^{2}\right)$ with $b_{i} \in \mathbb{R}$ and that $a_{0}\left(2 a_{0}^{2}-c\right)=18\left(b_{1}^{2}+b_{2}^{2}\right)$. Therefore if $c>0$, then either $-\sqrt{\frac{c}{2}} \leq a_{0}<0$ or $\sqrt{\frac{c}{2}} \leq a_{0}$. Thus, $a\left(\Sigma_{c}^{1}\right)$ and hence $\Sigma_{c}^{1}$ is disconnected for $c>0$.

One can show that these are the only components, i.e. $\Sigma_{c}^{1}$ is connected if $c<0$ and has two components $\Sigma_{c}^{1, \pm}$ if $c>0$ where \pm stands for the sign of $a_{0}$ in the notation above.

We will refer to the regular parts of the level sets of $R_{c}$ and to the manifold parts of the sets $\Sigma_{c}$ as strata.

For the ranks of $K_{*}$ we have:

- $\operatorname{rank}\left(K_{*}(u)\right)=4$ for all $u \in F$ with $K(u) \in \Sigma_{c}$ and $c \neq 0$.

- $\operatorname{rank}\left(K_{*}(u)\right)=4$ for all $u \in F$ with $K(u) \in \Sigma_{0} \backslash \Sigma_{0}^{0}$,

- $\operatorname{rank}\left(K_{*}(u)\right)=2$ for all $u \in F$ with $K(u) \in \Sigma_{0}^{0} \backslash\{0\}$,

- $\operatorname{rank}\left(K_{*}(u)\right)=0$ for all $u \in F$ with $K(u)=0$.

Theorem 3.7. The image of the map $K: F \rightarrow V$ lies always in a single stratum. In particular, the differential $K_{*}$ has constant rank on $F$, and $\operatorname{rank}\left(K_{*}\right) \in$ $\{0,2,4,6\}$.

We start with the following lemma whose proof is left to the reader:

Lemma 3.8. Any two points in $F$ can be joined by a piecewise differentiable path $\gamma$ such that $\gamma^{\prime}(t)= \pm X_{i}$ or $\gamma^{\prime}(t)= \pm Y_{i}$ for some $i$, wherever $\gamma^{\prime}$ is defined.

Proof of theorem. We define vector fields $\bar{X}_{0}, \ldots, \bar{X}_{3}, \bar{Y}_{1}, \bar{Y}_{2}, \bar{Y}_{3}$ on $V$ by

$$
\left(\bar{X}_{0}, \ldots, \bar{X}_{3}, \bar{Y}_{1}, \bar{Y}_{2}, \bar{Y}_{3}\right)^{t}=K_{*}\left(\frac{\partial}{\partial a_{0}}, \frac{\partial}{\partial a_{1}}, \frac{\partial}{\partial a_{2}}, \frac{\partial}{\partial b_{0}}, \ldots, \frac{\partial}{\partial b_{3}}\right)^{t} \text {. }
$$

Thus if $\gamma$ is an integral curve of $X_{i}$ ( $Y_{i}$ resp.) in $F$ then the curve $K(\gamma)$ is an integral curve of $\overline{X_{i}}$ ( $\overline{Y_{i}}$ resp.) in $V$. Now one observes that these integral curves always stay inside the same stratum in $V$. This together with Lemma 3.8 finishes the proof.

Of course, if $K(F)=\{0\}$ then the connection form $\phi$ is flat, i.e. the holonomy group is not equal to $\mathrm{H}_{3}$.

Note that the stratum containing $K(F)$ is an invariant of the homothety class of $F$, hence indeed an invariant of the $\mathrm{H}_{3}$-connection. Therefore, we will refer to this stratum as the type of the $\mathrm{H}_{3}$-connection. E.g. we will talk about connections of type $\Sigma_{c}^{1,+}$ etc.

\section{NONCOMPLETENESS}

The purpose of this section is to prove the

Theorem 4.1. $H_{3}$-connections are never complete.

Proof. Let $M$ be a manifold with an $H_{3}$-connection $\nabla$, and let $(\pi, F, M, \omega$, $\phi, a, b, c)$ be an associated solution structure over $M$. By [KN, III.6.3.], the geodesics on $M$ are precisely those curves $\gamma$ in $M$ with the property that if $\tilde{\gamma}$ is a horizontal lift of $\gamma$ then $\omega\left(\tilde{\gamma}^{\prime}\right) \in V_{3}$ is constant. 
Now suppose that $\nabla$ is complete. Let $\tilde{\gamma}$ be an integral curve of the vector field $X_{1}$ in $F$ which projects down to the geodesic $\gamma=\pi(\tilde{\gamma})$ in $M$ and therefore by hypothesis is defined on all of $\mathbb{R}$. From the structure equations we get that $X_{1}\left(a_{2}\right)=6 b_{3}$ and $X_{1}\left(b_{3}\right)=a_{2}^{2}$, hence $6 a_{2}$ satisfies on $\tilde{\gamma}$ the differential equation

$$
y^{\prime \prime}=y^{2} \text {. }
$$

We shall see that the only solution of $(*)$ which is defined on all of $\mathbb{R}$ is $y=0$ and therefore $a_{2}=0$ along $\tilde{\gamma}$.

Since $\tilde{\gamma}$ was an arbitrary integral curve of $X_{1}$ we conclude that $a_{2} \equiv 0$ on $F$. But then the structure equations easily imply that $a=b \equiv 0$ on $F$ which means that the connection is flat, hence not an $\mathrm{H}_{3}$-connection, and this contradiction will finish the proof.

It remains to show that $(*)$ has no nontrivial solution. Note that any global solution $y$ is convex and hence either constant or unbounded. Suppose that $y$ is a global nonconstant solution, i.e. $y^{\prime}\left(t_{0}\right) \neq 0$ for some $t_{0}$. Replacing $y(t)$ by $y(-t)$ if necessary, we may assume that $y^{\prime}\left(t_{0}\right)>0$ and hence $y^{\prime}(t) \geq y^{\prime}\left(t_{0}\right)>0$ for all $t \geq t_{0}$.

We get

$$
\frac{d}{d t}\left(\left(y^{\prime}\right)^{2}-\frac{2}{3} y^{3}\right)=0
$$

hence

$$
\left(y^{\prime}\right)^{2}=C+\frac{2}{3} y^{3}
$$

for some constant $C$.

As $y$ is unbounded, i.e. $\lim _{t \rightarrow \infty} y=\infty$, we may assume that $y(t)^{3}>3|C|$ for all $t \geq t_{0}$ by increasing $t_{0}$ if necessary. Then we get for all $t \geq t_{0}$

$$
\left(y^{\prime}\right)^{2}>\frac{1}{3} y^{3}
$$

which implies

$$
\left(y^{-\frac{1}{2}}\right)^{\prime}<-\frac{1}{2 \sqrt{3}} .
$$

Thus we get for all $t \geq t_{0}$

$$
y(t)^{-\frac{1}{2}}<C_{1}-\frac{1}{2 \sqrt{3}} t
$$

for some constant $C_{1}$. But this is a contradiction as the left-hand side of this inequality is always positive whereas the right-hand side is negative for large $t$, and this finishes the proof.

\section{THE SINGULAR $H_{3}$-CONNECTIONS}

5.1 Immersions of solution structures. We will begin with the following

Definition 5.1. We call $(F, \omega, \phi, a, b, c)$ a pseudo solution structure if

(1) $F$ is a 7-dimensional connected manifold, $\omega+\phi$ is a coframe on $F$ with values in $V_{3} \oplus \mathfrak{h}_{3}, a, b$ are functions on $F$ with values in $V_{2}, V_{3}$ 
resp. and the structure equations (5)-(9) are satisfied for some constant $c \in \mathbb{R}$.

(2) there is a locally free $\operatorname{Sl}(2, \mathbb{R})$-action on $F$ such that if we define vector fields $E_{i}^{*}$ by $\left(E_{i}^{*}\right)_{p}:=\left.\frac{d}{d t}\right|_{t=0}\left(e^{t E_{i}}(p)\right)$ with the basis $\left\{E_{i}\right\}$ of $\mathfrak{h}_{3}$ as before, then $\phi\left(E_{i}^{*}\right)=E_{i}$ for all $i$.

Consider the canonical projection $\pi: F \rightarrow s(2, \mathbb{R}) \backslash F=: M$. In general, $M$ will not be a manifold. We shall call a pseudo solution structure holonomic over $M$ if the $S l(2, \mathbb{R})$-action is globally free and $M$ is a manifold. In this case, $(\pi, F, M, \omega, \phi, a, b, c)$ is a solution structure.

Definition 5.2. Let $(F, \omega, \phi, a, b, c)$ a pseudo solution structure. A (local) vector field $S$ on $F$ is called a (local) infinitesimal symmetry of $F$ if $\mathfrak{L}_{S}(\omega)=$ $\mathfrak{L}_{S}(\phi)=0$.

Proposition 5.3. The local infinitesimal symmetries on $F$ form a Lie algebra $\mathfrak{g} \subseteq \mathfrak{X}(F)$ whose dimension equals the corank of $K_{*}$ where $K:=a+b: F \rightarrow V$. In fact, they span $\operatorname{ker}\left(K_{*}\right)$ at each point $p \in F$.

Proof. It is immediate that infinitesimal symmetries are closed under Lie brackets and hence form a Lie algebra. Next, any $S \in \mathfrak{g}$ vanishes either everywhere or nowhere. To see this note that along an integral curve $\gamma$ of $X_{i}$ ( $Y_{i}$ resp.), $\left[S, \gamma^{\prime}\right] \equiv 0$, hence $S$ vanishes either everywhere or nowhere along $\gamma$. Then apply Lemma 3.8 .

Moreover, it is obvious from the structure equations that $K_{*}(S)=0$ for all $S \in \mathfrak{g}$, hence $\operatorname{dim}(\mathfrak{g}) \leq \operatorname{corank}\left(K_{*}\right)$.

It remains to show that any tangent vector $S_{p} \in \operatorname{ker}\left(K_{*}\right)$ at some point $p \in F$ extends to an infinitesimal symmetry. This follows essentially from the uniqueness result of solutions to the structure equations (cf. [Br2] for details).

Of course this means that every $H_{3}$-connection admits at least one 1-parameter family of (local) symmetries. In fact, the (local) symmetry group $G$ of $M$ is a Lie group whose dimension equals the corank of $K$.

Definition 5.4. Let $(F, \omega, \phi, a, b, c)$ be a pseudo solution structure.

(1) $F$ is called saturated if the map $K: F \rightarrow K(F) \subseteq V$ is a principal $G$ fibration where $G$ denotes the symmetry group of $F$. This is equivalent to saying that every local symmetry of $F$ extends to a global symmetry.

(2) $F$ is called maximal if it is saturated and $K(F)$ is an entire stratum.

The following proposition can be shown using the standard techniques to prove generalizations of the second Cartan lemma (cf. e.g. $[\mathrm{Br} 2])$. We will leave the proof to the reader.

Proposition 5.5. Let $(\pi, F, M, \omega, \phi, a, b, c)$ be a solution structure over some manifold $M$ and suppose that $F$ is saturated. Let $\left(\pi^{\prime}, F^{\prime}, M^{\prime}, \omega^{\prime}, \phi^{\prime}, a^{\prime}, b^{\prime}, c^{\prime}\right)$ be another solution structure over a simply connected manifold $M^{\prime}$ such that $K\left(F^{\prime}\right) \subseteq K(F)$. Then there exist immersions $\jmath: F^{\prime} \rightarrow F$ and $\imath: M^{\prime} \rightarrow M$ such 
that $\jmath^{*}\left(\omega^{\prime}+\phi^{\prime}\right)=\omega+\phi, a^{\prime}+b^{\prime}=(a+b) \circ \jmath$ and such that the diagram

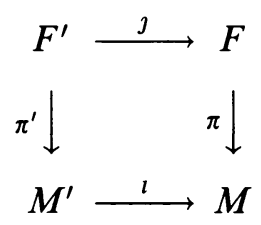

commutes. In particular, $l$ is connection preserving, i.e. $l_{*}\left(\nabla_{X}^{\prime} Y\right)=\nabla_{l_{*}(X)} l_{*}(Y)$ for all vector fields $X$ and $Y$ on $M^{\prime}$, where $\nabla, \nabla^{\prime}$ denote the covariant derivatives on $M, M^{\prime}$ resp.

Therefore, if we have a maximal solution structure then every other simply connected $H_{3}$-manifold of the same type can be immersed into $M$ by a connection preserving map. It also illustrates the significance of the examples given in $\S 2$ which will be discussed now.

Let us first determine the subset of an $\mathrm{H}_{3}$-manifold on which the symmetry group of the connection acts locally free.

Lemma 5.6. Let $M$ be an $H_{3}$-manifold and let $U \subseteq M$ be the set on which the local symmetries of $M$ act fixed point free or-equivalently-where the infinitesimal symmetries of $M$ do not vanish. Let $W^{\text {reg }} \subseteq V$ be the points in $V=V_{2} \oplus V_{3}$ on which the group $\operatorname{Sl}(2, \mathbb{R})$ acts locally free, i.e. $W^{\mathrm{reg}}$ is the union of all regular $\operatorname{Sl}(2, \mathbb{R})$ orbits of $V$. Then

$$
U=\pi\left(K^{-1}\left(W^{\mathrm{reg}}\right)\right) \text {. }
$$

In particular, $U$ is an open dense subset of $M$.

Proof. For $p \in M$, the infinitesimal symmetries on $M$ do not vanish at $p$ iff the infinitesimal symmetries on $F$ do not lie in the kernel of $\pi_{*}$ at all points $x \in \pi^{-1}(p)$. If we let $Y_{1}, Y_{2}, Y_{3}$ denote the vertical vector fields of the canonical frame of $F$, then this is equivalent to saying that $K_{*}\left(Y_{i}\right)_{x} \neq 0$ for all $x \in \pi^{-1}(p)$. Finally, the $S l(2, \mathbb{R})$-equivariance of $K$ implies that this is satisfied iff $K(x) \in W^{\text {reg }}$ for all $x \in \pi^{-1}(p)$. Now $W^{\text {reg }}$ is open dense in $V$, hence so is $U$ in $M$.

We now consider the examples from $\S 2$. For each one of them, the structure polynomials $a, b$ as well as the constant $c$ were given. These polynomials gave the restriction of the map $K: F \rightarrow V$ to the section of $F$ given by the particular (co)frame. Therefore, the image set $K(F)$ equals the $S l(2, \mathbb{R})$-orbit of the structure polynomials in each example.

(1) In Example 2.1, we have the structure polynomials $a=x^{2}, b=\frac{1}{3} x^{3}$ and $c=0$. Thus,

$$
K(F)=S l(2, \mathbb{R})(a, b)=\left\{\left(u^{2}, \frac{1}{3} u^{3}\right) \mid u \neq 0\right\}=\Sigma_{0}^{0} \backslash\{0\} .
$$

Also, it is not hard to show that the map $K$ is a principal $A S l(2, \mathbb{R})$ fibration and hence $F$ is maximal. Thus, we have a maximal solution structure of type $\Sigma_{0}^{0}$ and from Proposition 5.5 we get

Corollary 5.7. Let $M$ be a connected 4-manifold with an $H_{3}$-connection $\nabla$ of type $\Sigma_{0}^{0}$ and let $\tilde{M}$ denote its universal cover with the induced connection $\tilde{\nabla}$. Then there exists a connection preserving immersion

$$
\imath: \tilde{M} \rightarrow G / H
$$


with $G / H$ as in $\S 2.1$, i.e. $l_{*}\left(\tilde{\nabla}_{X} Y\right)=\bar{\nabla}_{l_{*}(X) l_{*}}(Y)$ for all vector fields $X, Y$ on $M$, where $\bar{\nabla}$ denotes the connection on $G / H$.

(2) Consider the connections on $M_{0}^{ \pm}$described in $\S 2.2$. The structure polynomials in these cases are $(a, b) \varphi_{0}^{ \pm}\left(t_{0} y, x\right)$ and hence

$$
K(F)=S l(2, \mathbb{R})(a, b)=\varphi_{0}^{ \pm}\left\{(u, v) \in V_{1} \times V_{1} \mid\langle u, v\rangle_{1} \neq 0 .\right\}
$$

which is precisely $\left(\Sigma_{0}^{ \pm} \backslash \Sigma_{0}^{0}\right) \cap W^{\text {reg }}$. Again, we can show that the map $K$ is a principal $G$-fibration where $G=N_{3}$ is the 3-dimensional Heisenberg group which acts via symmetries on $M_{0}^{ \pm}$. Hence $F$ is saturated. We will show that there exist maximal solution structures of types $\Sigma_{0}^{ \pm}$ and they therefore contain $M_{0}^{ \pm}$as the dense open subset on which the symmetry group acts locally free.

(3) Consider the connections on $M_{c}^{ \pm}$described in $\S 2.2$ and suppose that $c \neq 0$. The structure polynomials in these cases are

$$
(a, b)=\left( \pm x^{2}+\frac{1}{2}\left(2 t_{0}^{2} \pm c\right) y^{2}, t_{0} y\left(\mp x^{2}+\frac{2 t_{0}^{2} \pm c}{6} y^{2}\right)\right)
$$

which is seen to lie in $\Sigma_{c}^{1}$. We find that

$$
\Sigma_{c}^{1} \cap W^{\mathrm{reg}}=\left\{(a, b) \in \Sigma_{c}^{1} \mid\left\langle\langle b, b\rangle_{2},\langle b, b\rangle_{2}\right\rangle_{2} \neq 0\right\} .
$$

Consider first the case $c>0$. Then recall that $\Sigma_{c}^{1}$ has two components, and we check that $M_{c}^{ \pm}$is of type $\Sigma_{c}^{1, \pm}$ if $c>0$. Moreover, in each case $K\left(F_{c}^{ \pm}\right)=\Sigma_{c}^{1}, \pm \cap W^{\text {reg }}$ where $F_{c}^{ \pm}$is the solution structure associated to $M_{c}^{ \pm}$. We also check that $F_{c}^{ \pm}$is saturated and therefore, as a consequence of Proposition 5.5 we have

Corollary 5.8. Let $M$ be a connected 4-manifold with an $H_{3}$-connection $\nabla$ of type $\Sigma_{c}^{1, \pm}$ with $c>0$. Let $U \subseteq M$ be the subset on which the symmetry group of $M$ acts locally free and let $U=\bigsqcup_{i} U_{i}$ be the decomposition of $U$ into its connected components. Then there exist connection preserving immersions $\imath: \tilde{U}_{i} \rightarrow M_{c}^{ \pm}$with $M_{c}^{ \pm}$from $\S 2.2$, where $\tilde{U}_{i}$ denotes the universal cover of $U_{i}$.

Now assume $c<0$. In this case, both examples $M_{c}^{ \pm}$have type $\Sigma_{c}^{1}$. One checks that $\Sigma_{c}^{1} \cap W^{\text {reg }}$ has two connected components, and they equal $K\left(F_{c}^{+}\right) \sqcup K\left(F_{c}^{-}\right)$with $F_{c}^{ \pm}$as above. Again, we check that $F_{c}^{ \pm}$is saturated, hence Proposition 5.5 yields

Corollary 5.9. Let $M$ be a connected 4-manifold with an $H_{3}$-connection $\nabla$ of type $\Sigma_{c}^{1}$ with $c<0$. Let $U \subseteq M$ be the subset on which the symmetry group of $M$ acts locally free and let $U=\bigsqcup_{i} U_{i}$ be the decomposition of $U$ into its connected components. Then there exist connection preserving immersions

$$
\imath: \tilde{U}_{i} \rightarrow M_{c}^{+} \sqcup M_{c}^{-}
$$

with $M_{c}^{ \pm}$from $\S 2.2$, where $\tilde{U}_{i}$ denotes the universal cover of $U_{i}$.

The difference between these two results is that in the case $c>0$ an $H_{3}$-connection of type $\Sigma_{c}^{1, \pm}$ on $M$ is either locally equivalent to $M_{c}^{+}$ or locally equivalent to $M_{c}^{-}$since these connections are of different type. 
In the case $c<0$, however, it is very well possible that an $\mathrm{H}_{3}$ connection is locally equivalent to $M_{c}^{+}$in some neighborhood, but equivalent to $M_{c}^{-}$in some other neighborhood on the same manifold $M$. In fact, this will happen if a maximal solution structure of type $\Sigma_{c}^{1}$ exists.

Also, we can conclude that connections of type $\Sigma_{c}^{1,+}$ with $c>0$ have (local) symmetry group $S U(2)$, and connections of type $\Sigma_{c}^{1,-}$ with $c>0$ or of type $\Sigma_{c}^{1}$ with $c<0$ have (local) symmetry group $\operatorname{Sl}(2, \mathbb{R})$.

(4) Consider the connections on $M_{k}^{ \pm}$described in $\S 2.3$ for $k \neq 0$. The structure polynomials in these cases are

$$
(a, b)=\left(u^{2}+v, \frac{1}{3} u\left(u^{2}-3 v\right)\right)
$$

where $u=k t_{0} y$ and $v=k\left(x^{2} \pm y^{2}\right)$. Also, the structure constant $c=$ $\pm 6 k^{2}$. Thus, $M_{k}^{ \pm}$is of type $\Sigma_{c}^{2}$. Note that $\Sigma_{c}^{2} \cap W^{\text {reg }}=\left\{\varphi_{c}^{2}(u, v) \mid u\right.$ does not divide $v$ \}.

By an analysis similar to the previous case, we obtain from Proposition 5.5

Corollary 5.10. Let $M$ be a connected 4-manifold with an $H_{3}$-connection $\nabla$ of type $\Sigma_{c}^{2, \pm}$ with $c>0$ and let $k$ such that $\operatorname{sign}(k)= \pm$ and $c=6 k^{2}$. Let $U \subseteq M$ be the subset on which the symmetry group of $M$ acts locally free and let $U=\bigsqcup_{i} U_{i}$ be the decomposition of $U$ into its connected components. Then there exist connection preserving immersions $\imath: \tilde{U}_{i} \rightarrow M_{k}^{+}$with $M_{k}^{+}$from $\S 2.3$, where $\tilde{U}_{i}$ denotes the universal cover of $U_{i}$.

Corollary 5.11. Let $M$ be a connected 4-manifold with an $H_{3}$-connection $\nabla$ of type $\Sigma_{c}^{2}$ with $c<0$ and let $k$ such that $c=-6 k^{2}$. Let $U \subseteq M$ be the subset on which the symmetry group of $M$ acts locally free and let $U=\bigsqcup_{i} U_{i}$ be the decomposition of $U$ into its connected components. Then there exist connection preserving immersions

$$
\imath: \tilde{U}_{i} \rightarrow M_{k}^{-} \sqcup M_{-k}^{-}
$$

with $M_{ \pm k}^{-}$from $\S 2.3$, where $\tilde{U}_{i}$ denotes the universal cover of $U_{i}$.

The difference between these two results is as in the previous case.

Also, we can conclude that connections of type $\Sigma_{c}^{2,+}$ with $c>0$ have (local) symmetry group $S U(2)$, and connections of type $\Sigma_{c}^{2,-}$ with $c>0$ or of type $\Sigma_{c}^{2}$ with $c<0$ have (local) symmetry group $\operatorname{Sl}(2, \mathbb{R})$.

Of course, these corollaries are not ideal statements. The main question which they answer only partially is

For which (connected) strata do maximal pseudo solution structures exist? If they do, are they holonomic?

We already answered these questions affirmatively for $\mathrm{H}_{3}$-connections of type $\Sigma_{0}^{0}$ and will also obtain an affirmative answer for the types $\Sigma_{0}^{ \pm}$. In the remaining cases, however, the above statements are the best we can do.

5.2 Reductions of $\mathrm{H}_{3}$-connections. We will now construct further reductions of the $\mathfrak{h}_{3}$-reductions of singular $H_{3}$-connections. These reductions are equipped 
with another connection with a 1-dimensional Holonomy group. However, these connections will have torsion.

5.2.1 $H_{3}$-connections of type $\Sigma_{0}^{0}$. Let $M$ be a connected 4-manifold with an $H_{3}$-connection $\nabla$ of type $\Sigma_{0}^{0}$. This means that we have an $\mathfrak{h}_{3}$-reduction $F$ of the total coframe bundle $\mathfrak{F}$ of $M$ and an $S l(2, \mathbb{R})$-equivariant map $K: F \rightarrow$ $\Sigma_{0}^{0} \backslash\{0\} \subseteq V$.

We define functions $r_{1}$ and $r_{2}$ by $K(u)=\varphi_{0}^{0}\left(r_{1}(u) x+r_{2}(u) y\right)$ where $\varphi_{0}^{0}$ is the map defined in $\S 3$. Then the structure equations imply that

$$
\begin{aligned}
& d r_{1}=3 r_{2}^{2} \omega_{0}-2 r_{1} r_{2} \omega_{1}+r_{1}^{2} \omega_{2}+2 r_{2} \phi_{0}-r_{1} \phi_{1}, \\
& d r_{2}=\quad r_{2}^{2} \omega_{1}-2 r_{1} r_{2} \omega_{2}+3 r_{1}^{2} \omega_{3}+r_{2} \phi_{1}-2 r_{1} \phi_{2} .
\end{aligned}
$$

$\operatorname{rank}\left(K_{*}\right)=2$, and therefore $K$ is a submersion. Since $\operatorname{Sl}(2, \mathbb{R})$ acts transitively on $\Sigma_{0}^{0} \backslash\{0\}$ it follows that $K$ is surjective. $\bar{F}:=K^{-1}\left(\varphi_{0}^{0}(x)\right)$ is a 5dimensional submanifold of $F$ which intersects all fibers $\pi^{-1}(p), p \in M$, and the intersection with each fiber is connected; for $\pi\left(u_{1}\right)=\pi\left(u_{2}\right)$ iff $u_{1}=L_{h}\left(u_{2}\right)$ for some $h \in \bar{H}$ where

$$
\bar{H}:=\left\{\rho_{3}\left(\begin{array}{ll}
1 & 0 \\
t & 1
\end{array}\right) \mid t \in \mathbb{R}\right\} \subseteq H_{3}
$$

which is connected. It follows that $\bar{F}$ is a principal $\bar{H}$-bundle.

Let $l: \bar{F} \hookrightarrow F$ be the inclusion map. We compute that the 1 -forms $\bar{\omega}_{0}, \ldots$, $\bar{\omega}_{3}, \bar{\phi}$ with $\bar{\omega}_{i}:=l^{*}\left(\omega_{i}\right)$ and $\bar{\phi}:=l^{*}\left(\phi_{3}+\frac{1}{2} \omega_{1}\right)$ yield a coframe of $\bar{F}$, satisfying

$$
\begin{array}{rlrl}
d \bar{\omega}_{0}= & 3 \bar{\omega}_{0} \wedge \bar{\omega}_{2} & -2 \bar{\omega}_{1} \wedge \bar{\phi}, \\
d \bar{\omega}_{1}= & 9 \bar{\omega}_{0} \wedge \bar{\omega}_{3}+3 \bar{\omega}_{1} \wedge \bar{\omega}_{2}-4 \bar{\omega}_{2} \wedge \bar{\phi}, \\
d \bar{\omega}_{2}= & 9 \bar{\omega}_{1} \wedge \bar{\omega}_{3} & -6 \bar{\omega}_{3} \wedge \bar{\phi}, \\
d \bar{\omega}_{3}= & 6 \bar{\omega}_{2} \wedge \bar{\omega}_{3}, & \\
d \bar{\phi}= & -\frac{9}{2}\left(3 \bar{\omega}_{0} \wedge \bar{\omega}_{3}-\bar{\omega}_{1} \wedge \bar{\omega}_{2}\right) .
\end{array}
$$

Let $\sigma$ be the (up to multiples) unique parallel symplectic form on $M$. One computes that

$$
\pi^{*}(\sigma)=3 \bar{\omega}_{0} \wedge \bar{\omega}_{3}-\bar{\omega}_{1} \wedge \bar{\omega}_{2}=-\frac{2}{9} d \bar{\phi}
$$

and-using that the restriction $\pi: \bar{F} \rightarrow M$ is a homotopy equivalence-we arrive at the

Proposition 5.12. Let $M$ be an $H_{3}$-manifold of type $\Sigma_{0}^{0}$, and let $\sigma$ be the parallel symplectic form on $M$. Then $\sigma$ is exact.

We denote the frame dual to the coframe on $\bar{F}$ by $X_{0}, \ldots, X_{3}, Y$. We have $\mathfrak{L}_{Y}(\phi)=0$, and this implies that $\phi$ is the connection form of a connection on the principal bundle $\pi: \bar{F} \rightarrow M$. Its curvature is given by the symplectic form $\sigma$. However, this connection has torsion.

5.2.2 $H_{3}$-connections of type $\Sigma_{0}^{ \pm}$. Let $M$ be a connected 4-manifold with an $H_{3}$-connection $\nabla$ of type $\Sigma_{0}^{ \pm}$. This means that we have an $\mathfrak{h}_{3}$-reduction $F$ of the total coframe bundle $\mathfrak{F}$ of $M$ and an $S l(2, \mathbb{R})$-equivariant map $K: F \rightarrow$ $\Sigma_{0}^{ \pm} \backslash \Sigma_{0}^{0} \subseteq V$.

Recall the double covering $\varphi_{0}^{ \pm}: P^{ \pm} \rightarrow \Sigma_{0}^{ \pm} \backslash \Sigma_{0}^{0}$ defined in $\S 3$. Here, $P^{+}=$ $\left\{(u, v) \in V_{1} \times V_{1} \mid v \neq 0\right.$ and $\left.v^{2} \neq 3 u^{2}\right\}$ and $P^{-}=\left\{(u, v) \in V_{1} \times V_{1} \mid v \neq 0\right\}$. 
Let $P_{0}^{ \pm}:=\left\{u \in V_{1} \mid(u, x) \in P^{ \pm}\right\}$, i.e. $P^{+}=V_{1} \backslash\left\{ \pm \frac{1}{\sqrt{3}} x\right\}$ and $P^{-}=V_{1}$. Let $\bar{F}:=K^{-1}\left(\left\{\varphi_{0}^{ \pm}(u, x) \mid u \in P_{0}^{ \pm}\right\}\right) \subseteq F$.

Since $\varphi_{0}^{ \pm}$is a double cover and $K$ has constant maximal rank it follows that $\bar{F}$ is a smooth 5 -dimensional submanifold of $F$. Moreover, the $S l(2, \mathbb{R})$ equivariance of $K$ implies that $\bar{F}$ is a reduction of $F$ with structure group

$$
\left\{\left(\begin{array}{ll}
1 & 0 \\
t & 1
\end{array}\right) \mid t \in \mathbb{R}\right\} \subseteq S l(2, \mathbb{R})
$$

In particular, $\bar{F}$ is connected. We then define a function $r: \bar{F} \rightarrow P_{0}^{ \pm}$by the equation

$$
K(u)=\varphi_{0}^{ \pm}(r(u), x) \quad \text { for all } u \in \bar{F} .
$$

Let $l: \bar{F} \hookrightarrow F$ be the inclusion map. We compute that the 1 -forms $\bar{\omega}_{0}, \ldots$, $\bar{\omega}_{3}, \bar{\phi}$ with $\bar{\omega}_{i}:=l^{*}\left(\omega_{i}\right)$ and $\bar{\phi}:=l^{*}\left(\phi_{3}-3 r_{2} \omega_{0}+r_{1} \omega_{1}\right)$ yield a coframe of $\bar{F}$, satisfying

$$
\begin{array}{rlr}
d \bar{\omega}_{0}= & 12 r_{2} \bar{\omega}_{0} \wedge \bar{\omega}_{1}-6 r_{1} \bar{\omega}_{0} \wedge \bar{\omega}_{2} & -2 \bar{\omega}_{1} \wedge \bar{\phi}, \\
d \bar{\omega}_{1}= & 18 r_{2} \bar{\omega}_{0} \wedge \bar{\omega}_{2}-18 r_{1} \bar{\omega}_{0} \wedge \bar{\omega}_{3}-6 r_{1} \bar{\omega}_{1} \wedge \bar{\omega}_{2}-4 \bar{\omega}_{2} \wedge \bar{\phi}, \\
d \bar{\omega}_{2}= & 18 r_{2} \bar{\omega}_{0} \wedge \bar{\omega}_{3}+6 r_{2} \bar{\omega}_{1} \wedge \bar{\omega}_{2}-18 r_{1} \bar{\omega}_{1} \wedge \bar{\omega}_{3}-6 \bar{\omega}_{3} \wedge \bar{\phi}, \\
d \bar{\omega}_{3}=6 r_{2} \bar{\omega}_{1} \wedge \bar{\omega}_{3}-12 r_{1} \bar{\omega}_{2} \wedge \bar{\omega}_{3}, & \left.\bar{\omega}_{1} \wedge \bar{\omega}_{2}\right),
\end{array}
$$

where $r=r_{1} x+r_{2} y$, and the functions $r_{1}$ and $r_{2}$ satisfy

$$
\begin{aligned}
& d r_{1}=9 r_{2}^{2} \bar{\omega}_{0}-6 r_{1} r_{2} \bar{\omega}_{1}-s \bar{\omega}_{2} \quad+2 r_{2} \bar{\phi}, \\
& d r_{2}=\quad 3 r_{2}^{2} \bar{\omega}_{1}-6 r_{1} r_{2} \bar{\omega}_{2}-3 s \bar{\omega}_{3} \text {, }
\end{aligned}
$$

with $s=-3 r_{1}^{2} \pm 1$. Note that $r \in P_{0}^{ \pm}$implies that $r_{2}$ and $s$ cannot vanish simultaneously.

Like in the previous section-using that the restriction $\pi: \bar{F} \rightarrow M$ is a homotopy equivalence-we obtain the

Proposition 5.13. Let $M$ be an $H_{3}$-manifold of type $\Sigma_{0}^{ \pm}$, and let $\sigma$ be the parallel symplectic form on $M$. Then $\sigma$ is exact.

Again, we see that $\phi$ is the connection form of a connection on the principal bundle $\pi: \bar{F} \rightarrow M$ whose curvature is given by the symplectic form $\sigma$. As before, this connection has torsion.

We will now show the existence of maximal saturated holonomic solution structures of types $\Sigma_{0}^{ \pm}$which we mentioned earlier. Note that if such a structure exists then $r: \bar{F} \rightarrow P_{0}^{ \pm}$must be a principal $G$-fibration where $G$ is the symmetry group of the connection.

Recall Example $M_{0}^{ \pm}$from \$2.2. Performing the construction above we obtain a solution to equations (11) on the set $G \times U_{1}^{ \pm} \rightarrow U_{1}^{ \pm}$where $G$ is the 3dimensional Heisenberg group and $U_{1}^{ \pm}=\left\{u \in P_{0}^{ \pm} \mid r_{2} \neq 0\right\}$. In this case, $r: G \times U_{1}^{ \pm} \rightarrow U_{1}^{ \pm}$is simply projection onto the second factor.

We will now give a second explicit solution to the equations (11). This time, we will consider the set $G \times U_{2}^{ \pm}$with $G$ as before and $U_{2}^{ \pm}=\left\{u \in P_{0}^{ \pm} \mid s \neq 0\right\}$.

Let $\mathfrak{g}$ be the Lie algebra of $G$. On $G \times U_{2}^{ \pm}$we define the $\mathfrak{g}$-valued 1-form $\alpha:=p^{*}\left(\omega_{G}\right)$ where $\omega_{G}$ is the left invariant Maurer-Cartan form on $G$, and $p: G \times U_{2}^{ \pm} \rightarrow G$ is the projection map. We can decompose $\alpha$ into three real valued 1-forms $\alpha_{1}, \alpha_{2}, \alpha_{3}$ satisfying $d \alpha_{1}=d \alpha_{2}=0$ and $d \alpha_{3}=d \alpha_{1} \wedge d \alpha_{2}$. 
Then one can check that the following forms are a solution to the structure equations (11) on $G \times U_{2}^{ \pm}$:

$$
\begin{array}{lrrrr}
\bar{\omega}_{0}= & & - & -6 s \alpha_{3}, \\
\bar{\omega}_{1}= & & \frac{1 \pm 6 r_{1}^{2}-3 r_{1}^{4}}{s} \alpha_{1}- & \frac{24 r_{1}}{s} \alpha_{2}+36 r_{1} r_{2} \alpha_{3}, \\
\bar{\omega}_{2}= & \frac{2}{s} d r_{1} & -\frac{6 r_{1} r_{2}\left(r_{1}^{2} \mp 1\right)}{s} \alpha_{1}- & \frac{12 r_{2}}{s} \alpha_{2}+ & 18 r_{2}^{2} \alpha_{3}, \\
\bar{\omega}_{3}=-\frac{4 r_{1} r_{2}}{s^{2}} d r_{1}+\frac{2}{3 s} d r_{2}+ & r_{2}^{2} \alpha_{1}, & \\
\bar{\phi}= & \frac{6 r_{1}\left(1 \pm r_{1}^{2}\right)}{s} \alpha_{1}-\frac{6\left(9 r_{1}^{2} \pm 1\right)}{s} \alpha_{2} \pm & 36 r_{2} \alpha_{3} .
\end{array}
$$

$G$ acts on $G \times U_{2}^{ \pm}$in a canonical way from the left, and this action preserves $\omega$ and $r$, i.e. is an action by symmetries.

It follows now that these two solutions of (11) can be regarded as local trivializations of a principal $G$-bundle over $P_{0}^{ \pm}$. Of course, since $U_{2}^{-}=P_{0}^{-}$the second solution is already the entire bundle. On the other hand, for $P_{0}^{+}$we know that the bundle is oriented by the volume element $\bar{\omega}_{0} \wedge \cdots \wedge \bar{\phi}$. Therefore, the bundle must be trivial as well, hence in either case we conclude that there exists a solution to equations (11) on $G \times P_{0}^{ \pm}=: \bar{F}^{ \pm}$.

It is not hard to see that a solution to (11) completely determines a solution structure on $F^{ \pm}:=G \times\left(\Sigma_{0}^{ \pm} \backslash \Sigma_{0}^{0}\right)$, i.e. we can 'reverse' the reduction to $\bar{F}^{ \pm}$ described before. It remains to show that these solution structure are holonomic, i.e. the quotient space $M^{ \pm}:=S l(2, \mathbb{R}) \backslash F^{ \pm}$is a manifold.

This can be seen as follows. Let us denote the frame dual to $\bar{\omega}_{0}, \ldots, \bar{\omega}_{3}, \bar{\phi}$ by $X_{0}, \ldots X_{3}, Y$. Then the $\mathbb{R}$-action on $\bar{F}^{ \pm}$defined by the flow along the vector field $Y$ is proper. Thus, $\mathbb{R} \backslash \bar{F}^{ \pm}$is a manifold, and clearly $M^{ \pm}=\mathbb{R} \backslash \bar{F}^{ \pm}$ as desired.

As a result of our discussion we conclude:

Corollary 5.14. Let $M$ be a simply connected connected 4-manifold with an $\mathrm{H}_{3}$ connection $\nabla$ of type $\Sigma_{0}^{ \pm}$and let $M^{ \pm}$be the manifolds described above. Then there exists a connection preserving immersion $\imath: M \rightarrow M^{ \pm}$.

5.2.3 $H_{3}$-coninections of type $\Sigma_{c}^{2}$. Let $M$ be a connected 4-manifold with an $H_{3}$-connection $\nabla$ of type $\Sigma_{c}^{2}$ where $c \in \mathbb{R} \backslash\{0\}$. This means that we have an $\mathfrak{h}_{3}$ reduction $F$ of the total coframe bundle $\mathfrak{F}$ of $M$ and an $S l(2, \mathbb{R})$-equivariant map $K: F \rightarrow \Sigma_{c}^{2} \subseteq V$.

Recall the diffeomorphism $\varphi_{c}^{2}$ from $\S 3$. Recall also that $V_{2, c}$ has one or two connected components depending on the sign of $c$.

$V_{2, c}$ contains a polynomial $k\left(x^{2} \pm y^{2}\right)$, where “ \pm " $=\operatorname{sign}(c)$ and $k^{2}=\frac{1}{6}|c|$. In the case $c>0$ the choice of sign of $k$ determines the component $V_{2, c}^{ \pm}$.

If we let $\bar{F}_{k}:=K^{-1}\left(\left\{\varphi_{c}^{2}\left(u, k\left(x^{2} \pm y^{2}\right)\right) \mid u \in V_{1}\right\}\right) \subseteq F$ then $\bar{F}_{k}$ is a smooth 5-dimensional submanifold of $F$ and moreover a reduction of $F$ with structure group

$$
S O(2) \subseteq S l(2, \mathbb{R}) \text { if } c>0,
$$

and

$$
S O(1,1) \subseteq S l(2, \mathbb{R}) \quad \text { if } c<0
$$


We then define a function $r: \bar{F}_{k} \rightarrow V_{1}$ by the equation

$$
K(u)=\varphi_{c}^{2}\left(r(u), k\left(x^{2} \pm y^{2}\right)\right) \quad \text { for all } u \in \bar{F}_{k} .
$$

Let $l: \bar{F}_{k} \hookrightarrow F$ be the inclusion map. We compute that the 1 -forms $\bar{\omega}_{0}, \ldots, \bar{\omega}_{3}, \bar{\phi}$ with $\bar{\omega}_{i}:=l^{*}\left(\omega_{i}\right)$ and $\bar{\phi}:=\frac{1}{2} \imath^{*}\left(\phi_{2} \mp \phi_{3}-r_{1}\left(3 \omega_{3} \pm \omega_{1}\right)+\right.$ $\left.r_{2}\left( \pm 3 \omega_{0}+\omega_{2}\right)\right)$ yield a coframe of $\bar{F}_{k}$, satisfying

$$
\begin{array}{lrrr}
d \bar{\omega}_{0}= & 12 r_{2} \bar{\omega}_{0} \wedge \bar{\omega}_{1}-6 r_{1} \bar{\omega}_{0} \wedge \bar{\omega}_{2} & \pm & 2 \bar{\omega}_{1} \wedge \bar{\phi}, \\
d \bar{\omega}_{1}= & 18 r_{2} \bar{\omega}_{0} \wedge \bar{\omega}_{2}-18 r_{1} \bar{\omega}_{0} \wedge \bar{\omega}_{3} & -6 r_{1} \bar{\omega}_{1} \wedge \bar{\omega}_{2}+\left(-6 \bar{\omega}_{0} \pm 4 \bar{\omega}_{2}\right) \wedge \bar{\phi}, \\
d \bar{\omega}_{2}= & 18 r_{2} \bar{\omega}_{0} \wedge \bar{\omega}_{3}+6 r_{2} \bar{\omega}_{1} \wedge \bar{\omega}_{2} & -18 r_{1} \bar{\omega}_{1} \wedge \bar{\omega}_{3}+\left(-4 \bar{\omega}_{1} \pm 6 \bar{\omega}_{3}\right) \wedge \bar{\phi}, \\
d \bar{\omega}_{3}= & 6 r_{2} \bar{\omega}_{1} \wedge \bar{\omega}_{3}-12 r_{1} \bar{\omega}_{2} \wedge \bar{\omega}_{3} & - & 2 \bar{\omega}_{2} \wedge \bar{\phi}, \\
d \bar{\phi}= \pm 6 k\left(3 \bar{\omega}_{0} \wedge \bar{\omega}_{3}-\right. & \left.\bar{\omega}_{1} \wedge \bar{\omega}_{2}\right), &
\end{array}
$$

where $r=r_{1} x+r_{2} y$, and the functions $r_{1}$ and $r_{2}$ satisfy

$$
\begin{aligned}
& d r_{1}=3\left(3 r_{2}^{2} \mp k\right) \bar{\omega}_{0}-6 r_{1} r_{2} \bar{\omega}_{1}+\left(3 r_{1}^{2}-k\right) \bar{\omega}_{2} \quad \mp 2 r_{2} \bar{\phi}, \\
& d r_{2}=\quad\left(3 r_{2}^{2} \mp k\right) \bar{\omega}_{1}-6 r_{1} r_{2} \bar{\omega}_{2}+3\left(3 r_{1}^{2}-k\right) \bar{\omega}_{3}+2 r_{1} \bar{\phi} .
\end{aligned}
$$

As before, we get the

Proposition 5.15. Let $M$ be an $H_{3}$-manifold of type $\Sigma_{2}^{c}$, and let $\sigma$ be the parallel symplectic form on $M$.

(1) If $c>0$ then the element of $H^{2}(M, \mathbb{R})$ represented by $\sigma$ is a multiple of the Euler class of a circle bundle over $M$.

(2) If $c<0$ then $\sigma$ is exact.

Proof. The first case follows from the Gysin sequence for circle bundles.

In the second case, we may assume that $\bar{F}_{k}$ has two components by passing to a double cover of $M$ if necessary. The restriction of $\pi$ to one connected component of $\bar{F}_{k}$ is then a homotopy equivalence. Finally, one can show that for a double cover $\alpha: \tilde{M} \rightarrow M$, the induced map $\alpha^{*}: H^{*}(M, \mathbb{R}) \rightarrow H^{*}(\tilde{M}, \mathbb{R})$ is injective.

Again, we see that $\phi$ is the connection form of a connection on the principal bundle $\pi: \bar{F} \rightarrow M$ whose curvature is given by the symplectic form $\sigma$. As before, this connection has torsion.

Remark. Since the description of $\Sigma_{c}^{1}$ does not give a parametrization by an $S l(2, \mathbb{R})$-equivariant map as in the other cases, we cannot get a reduction of it as easily. Therefore, it seems more dificult to make any statement about its symplectic form.

Remark. We will now give a hint how we obtained the examples in $\S 2$. Given a stratum $\Sigma$, it is not hard to find a smooth curve in $\Sigma \cap W^{\text {reg }}$ which intersects every orbit exactly once. These curves are the parametrized structure polynomials $(a, b)$ given for each example. Once we have this curve, say $\alpha(t)$, we consider $S:=K^{-1}(\alpha)$ which is a smooth submanifold of $F$ and intersects every fiber of $\pi: F \rightarrow M$ at most once. This means that $S$ is the image of a smooth section on some subset $U \subseteq M$. Then we let $\bar{\omega}_{i}: l^{*}\left(\omega_{i}\right)$ for $0 \leq i \leq 3$ where $l: S \hookrightarrow F$ is the inclusion map. The structure equations yield equations for $d \bar{\omega}_{i}$ and these can be solved explicitly. 
5.2.4 Regular $H_{3}$-connections. In this section we will discuss those $H_{3}$ connections on $M$ for which the map $K: F \rightarrow V$ has maximal rank 6 .

Unfortunately, in this case the structure equations involved are so complex that they cannot be solved explicitly on an dense open subset of $M$. A (not very illuminating) description of the connection on some open subset, however, can be obtained.

Let $U:=\left\{p \in M \mid\langle a, a\rangle_{2}<0\right.$, and $a$ does not divide $b$ on $\left.\pi^{-1}(p)\right\}$. For $p \in U$ there is a unique coframe $u \in \pi^{-1}(p)$ such that

$$
b_{3}(u)=1, \quad a(u)=f(u) x y \quad \text { with } f(u)^{2}=-\langle a(u), a(u)\rangle_{2}>0 .
$$

Using this section, we can describe the connection w.r.t. some coordinate system on $U$ as follows where the constants $c$ and $R_{c}$ are given.

Let $x_{0}, \ldots, x_{3}$ be local coordinates and define the functions

$t_{0}=\frac{1}{x_{0}}, \quad t_{1}=\frac{x_{1}}{x_{0}^{2}}, \quad t_{2}=\frac{x_{1}^{2}}{x_{0}^{3}}+\frac{x_{2}}{x_{0}}, \quad$ and $\quad t_{3}=\frac{x_{1}^{3}}{x_{0}^{4}}+3 \frac{x_{1} x_{2}}{x_{0}^{2}}-x_{0}^{2}+c+\frac{1}{x_{0}} r$,

where

$$
r= \pm 2 \sqrt{x_{1}^{3}-x_{2}^{3}+c x_{1} x_{2}+R_{c}} .
$$

Furthermore, let

$$
s_{i}:=\frac{\partial}{\partial x_{0}}\left(x_{0} t_{i}\right) \text { for all } i .
$$

Then we define a frame as follows:

$$
\begin{aligned}
& X_{0}=3 x_{0} t_{0} \frac{\partial}{\partial x_{0}}, \\
& X_{1}=x_{0} t_{1} \frac{\partial}{\partial x_{0}}+t_{0} \bar{X}_{1}, \\
& X_{2}=-x_{0} t_{2} \frac{\partial}{\partial x_{0}}+2 t_{1} \bar{X}_{1}+\bar{X}_{2}, \\
& X_{3}=-3 x_{0} t_{3} \frac{\partial}{\partial x_{0}}+3 t_{2} \quad \bar{X}_{1}+3 x_{0} t_{1} \quad \bar{X}_{2}+\bar{X}_{3},
\end{aligned}
$$

with

$$
\begin{aligned}
& \bar{X}_{1}= \\
& \bar{X}_{2}=-r \frac{\partial}{\partial x_{1}} \quad-\frac{\partial u}{\partial x_{1}} \frac{\partial}{\partial x_{3}}, \\
& \bar{X}_{3}=2\left(3 x_{2}^{2}-c x_{1}\right) \frac{\partial}{\partial x_{1}}+2\left(3 x_{1}^{2}+c x_{2}\right) \frac{\partial}{\partial x_{2}},
\end{aligned}
$$

and where $u=u\left(x_{1}, x_{2}\right)$ is some function satisfying $\bar{X}_{3}(u) \equiv 1$.

This frame is defined on $\left\{\left(x_{0}, \ldots, x_{3}\right) \mid x_{0} \neq 0, x_{1}^{3}-x_{2}^{3}+c x_{1} x_{2}+R_{c}>0\right\}$ for the given constants $c$ and $R_{c}$.

The connection form is then given as

$$
\phi=\phi_{1} E_{1}+\phi_{2} E_{2}+\phi_{3} E_{3}
$$


where

$$
\begin{aligned}
& \phi_{1}=-\sum_{i=0}^{3} s_{i} \omega_{i}, \\
& \phi_{2}=-\sum_{i=0}^{3} i t_{i-1} \omega_{i}, \\
& \phi_{3}=\sum_{i=0}^{3}(3-i) t_{i+1} \omega_{i}
\end{aligned}
$$

and where $\omega_{0}, \ldots, \omega_{3}$ denotes the dual basis of $X_{0}, \ldots, X_{3}$.

One can check that this connection is indeed torsion free and is a regular $H_{3}$-connection. Note in particular that the 1-dimensional symmetry group is given as the flow along the vector field $\partial / \partial x_{3}$.

\section{6. $H_{3}$-CONNECTIONS ON COMPACT MANIFOLDS}

The purpose of this section is to show the

Theorem 6.1. There are no $H_{3}$-connections on compact 4-manifolds.

Proof. We will show that any compact 4-manifold $M$ with an $H_{3}$-connection must be of type $\Sigma_{0}^{0}$. This together with Proposition 5.12 will finish the proof since symplectic forms on compact manifolds cannot be exact.

Suppose $M$ is compact and has an $H_{3}$-connection, and let $\{\pi, F, M, a$, $b, c\}$ be an associated solution structure. Consider the function

$$
\begin{array}{ccc}
f: F & \longrightarrow \mathbb{R} \\
u & \longmapsto\langle a(u), a(u)\rangle_{2}=-\operatorname{discr}(a(u))
\end{array}
$$

Since $f$ is constant along the fibers of $F$ there is a unique function $\underline{f}$ : $M \rightarrow \mathbb{R}$ such that $\underline{f} \circ \pi=f$.

We shall now use that $\underline{f}$ must have both a maximum and a minimum on $M$. Therefore, we shall investigate the critical points of $\underline{f}$.

Using the structure equations (5)-(9) we find that

$$
d f=-4\left(3 b_{3},-b_{2}, b_{1}, 3 b_{0}\right)\left(\begin{array}{cccc}
3 a_{1} & -2 a_{0} & & \\
6 a_{2} & a_{1} & -4 a_{0} & \\
& 4 a_{2} & -a_{1} & -6 a_{0} \\
& & 2 a_{2} & -3 a_{1}
\end{array}\right)\left(\begin{array}{c}
\omega_{0} \\
\vdots \\
\omega_{3}
\end{array}\right)
$$

The determinant of the matrix in this equation is $9 f^{2}$. So if $u \in F$ is a critical point of $f$ and $f(u) \neq 0$ then $b(u)=0$. We wish to compute the Hessian of $\underline{f}$ at a critical point $p \in M$ w.r.t. some appropriate coframe.

If $\underline{f}(p)<0$ at a critical point $p \in M$ then there is a coframe $u \in \pi^{-1}(p)$ with $\bar{a}_{0}(u)=a_{2}(u)=0$ and $a_{1}(u) \neq 0$. We compute the Hessian w.r.t. this coframe as

$$
2\left(\begin{array}{ccc}
a_{1}\left(2 c+a_{1}^{2}\right) & a_{1}\left(2 c+a_{1}^{2}\right) & -9 a_{1}\left(2 c+3 a_{1}^{2}\right) \\
-9 a_{1}\left(2 c+3 a_{1}^{2}\right) &
\end{array}\right) .
$$


We see easily that this matrix has some negative eigenvalue regardless of the values of $a_{1}$ and $c$. Therefore, $f$ cannot have a negative minimum, and we conclude that $f \geq 0$.

Let $\mathscr{C}:=\left\{\bar{a} \in V_{2} \mid\langle a, a\rangle_{2} \geq 0\right\}$. We find that $\mathscr{C}=\mathscr{C}_{+} \cup \mathscr{C}_{-}$with $\mathscr{C}_{ \pm}=$ $\left\{ \pm\left(v_{1}^{2}+v_{2}^{2}\right) \mid v_{1}, v_{2} \in V_{1}\right\}$, and $\mathscr{C}_{+} \cap \mathscr{C}_{-}=\{0\}$. Moreover, $\mathscr{C}_{ \pm}$is invariant under the $S l(2, \mathbb{R})$-action on $V_{2}$.

If $\underline{f}(p)>0$ at a critical point $p \in M$ then there is a coframe $u \in \pi^{-1}(p)$ with $a_{1}(u)=0$ and $a_{0}(u)=a_{2}(u)$. We compute the Hessian w.r.t. this coframe as

$$
8\left(\begin{array}{cccc}
9 a_{0}^{3} & 0 & -3 a_{0}\left(5 a_{0}^{2}-c\right) & 0 \\
0 & a_{0}\left(13 a_{0}^{2}-2 c\right) & 0 & -3 a_{0}\left(5 a_{0}^{2}-c\right) \\
-3 a_{0}\left(5 a_{0}^{2}-c\right) & 0 & a_{0}\left(13 a_{0}^{2}-2 c\right) & 0 \\
0 & -3 a_{0}\left(5 a_{0}^{2}-c\right) & 0 & 9 a_{0}^{3}
\end{array}\right) .
$$

The characteristic polynomial of this matrix is $r(\lambda)^{2}$, where

$$
r(\lambda)=\lambda^{2}-16 a_{0}\left(11 a_{0}^{2}-c\right) \lambda-576 a_{0}^{2}\left(2 a_{0}^{2}-c\right)\left(6 a_{0}^{2}-c\right) .
$$

If $a(u) \in \mathscr{C}_{+} \backslash\{0\}$, i.e. $a_{0}(u)>0$, then $r$ has at least one positive root since either $r(0)<0$ or $r^{\prime}(0)<0$. Therefore, $\underline{f}$ cannot have a positive maximum in $\pi\left(a^{-1}\left(\mathscr{C}_{+} \backslash\{0\}\right)\right)$.

Similarly, if $a(u) \in \mathscr{C}_{-} \backslash\{0\}$, i.e. $a_{0}(u)<0$, then $r$ has at least one negative root since either $r(0)<0$ or $r^{\prime}(0)>0$. Therefore, $\underline{f}$ cannot have a positive minimum in $\pi\left(f^{-1}\left(\mathscr{C}_{-} \backslash\{0\}\right)\right)$.

Suppose that $\underline{f}(p)=0$ for some $p \in M$ and $a \neq 0$ on $\pi^{-1}(p)$. Since $f \geq 0, p$ is critical. There is a coframe $u \in \pi^{-1}(p)$ with $a(u)= \pm x^{2}$. From $d f(u)=0$ we conclude that $b(u)=\tilde{b} x^{3}$ for some $\tilde{b} \in \mathbb{R}$. Computing the Hessian of $f$ at $u$ we get

$$
8\left(\begin{array}{cccc}
0 & & & \\
& 0 & & \pm 3 c \\
& & \mp 2 c & -9\left(9 \tilde{b}^{2} \mp 1\right)
\end{array}\right)
$$

The characteristic polynomial of this matrix is

$$
\lambda(\lambda \pm 16 c)\left(\lambda^{2}+72\left(9 \tilde{b}^{2} \mp 1\right) \lambda-576 c^{2}\right)
$$

The Hessian must be positive semidefinite, i.e. this polynomial cannot have any negative root. It is easily seen that this is satisfied only if $a(u)=x^{2}, c=0$ and $\tilde{b}^{2} \leq \frac{1}{9}$.

We conclude that $f(u)=0$ implies $a(u) \in \mathscr{C}_{+}$.

Suppose now that $a(U) \subseteq \mathscr{C}_{+} \backslash\{0\}$ for some open set $U \subseteq F$. From the above we conclude that $f(U)=0$. It follows that $K(U) \subseteq\left\{\left(v^{2}, \tilde{b} v^{3}\right) \mid v \in\right.$ $\left.V_{1} \backslash\{0\}, \tilde{b} \in \mathbb{R}\right\}$. Since this set is 3 -dimensional we get $\operatorname{rank}\left(K_{*}\right) \leq 3$ on $U$. But then Theorem 3.7 implies that $M$ is of type $\Sigma_{0}^{0}$. In particular, $a(F) \subseteq \mathscr{C}_{+} \backslash\{0\}$.

Thus, either $M$ is of type $\Sigma_{0}^{0}$ or $a(F) \subseteq \mathscr{C}_{-}$.

But in the latter case we conclude that $a(u)=0$ for some $u \in F$. Since $a(F) \subseteq \mathscr{C}_{-}$, hence $a_{0}, a_{2} \leq 0$, this means that $u$ is a maximum for both $a_{0}$ 
and $a_{2}$, hence $d a_{0}(u)=d a_{2}(u)=0$ and therefore $b(u)=0$. Computing the Hessian of $a_{0}$ at $u$ yields the matrix

$$
\left(\begin{array}{cccc}
0 & & 6 c & \\
& -4 c & & \\
6 c & & 0 & \\
& & & 0
\end{array}\right)
$$

This matrix must be negative semidefinite which is satisfied iff $c=0$. Thus, $a(u)=b(u)=c=0$, i.e. $K_{*}(u)=0$, and again Theorem 3.7 implies that the connection is flat, violating our assumption. This contradiction shows that $M$ is of type $\Sigma_{0}^{0}$, and this finishes the proof.

\section{REFERENCES}

[A] D.V. Alekseevskii, Compact quaternion spaces, Funkcional Anal. i Priložen. 2 (1968), 11-20.

[Ber] M. Berger, Sur les groupes d'holonomie des variétés à connexion affine et des variétés Riemanniennes, Bull. Soc. Math. France 83 (1955), 279-330.

[BD] T. Bröcker and T. Dieck, Representations of compact Lie groups, Springer-Verlag, 1985.

[Br1] R. Bryant, Metrics with exceptional holonomy, Ann. of Math. 126 (1987), 525-576.

[Br2] - Two exotic holonomies in dimension four, path geometries, and twistor theory, Proc. Sympos. Pure Math., vol. 53, Amer. Math. Soc., Providence, R.I., 1991, pp. 33-88.

[EDS] R. Bryant, S. Chern, R. Gardner, H. Goldschmidt, and P. Griffith, Exterior differential systems, Springer-Verlag, 1991.

[C] E. Calabi, Métriques kähleriennes et fibrés holomorphes, Ann. Ecole. Norm. Sup. 12 (1979), 269-294.

[KN] S. Kobayashi and K. Nomizu, Foundations of differential geometry, vols. 1 and 2, Interscience, 1969.

[S] J. Simons, On transitivity of holonomy systems, Ann. of Math. 76 (1962), 213-234.

Department of Mathematics, Washington University, St. Louis, Missouri 63130

Current address: Max-Planck-Institut für Mathematik, Gottfried-Claren-Strasse 26, D-53225

Bonn, Germany

E-mail address: lorenz@math.wustl.edu 